\title{
In Situ Molecular Imaging of the Biofilm and Its Matrix
}

\author{
Yuanzhao Ding, ${ }^{\dagger} \neq, \S$ Yufan Zhou, ${ }^{\perp,}+$ Juan Yao, ${ }^{\S},{ }^{\ddagger}$ Craig Szymanski, ${ }^{\perp}$ James Fredrickson, ${ }^{\S}$ Liang \\ Shi, ${ }^{\S}$ Bin Cao, ${ }^{\dagger}, \|,{ }^{*}$ Zihua Zhu, ${ }^{\perp, *}$ and Xiao-Ying $\mathrm{Yu}^{\S, *}$ \\ ${ }^{\dagger}$ Singapore Centre for Environmental Life Sciences Engineering (SCELSE), Nanyang Technological University, \\ 637551, Singapore \\ ₹Interdisciplinary Graduate School (IGS), Nanyang Technological University (NTU), 639798, Singapore \\ ${ }^{\S}$ Earth and Biological Sciences Directorate, Pacific Northwest National Laboratory, Richland, WA 99354, USA \\ ${ }^{\perp}$ Environmental and Molecular Science Laboratory, Pacific Northwest National Laboratory, Richland, WA 99352, \\ USA \\ "School of Civil and Environmental Engineering, Nanyang Technological University (NTU), 639798, Singapore \\ $\$$ These authors contributed equally to this work.
}

* Correspondence and requests for materials should be addressed to X.-Y.Y. (email: xiaoying.yu@pnnl.gov),B.C. (email: bincao@ntu.edu.sg) or Z.Z. (email: zihua.zhu@pnnl.gov). 


\section{Supporting Information}

Supplemental information was provided to substantiate the discussion and provide additional experimental details including figures, tables, movies of 3D reconstructed images, and associated references.

\section{Experimental}

\section{ToF-SIMS}

ToF-SIMS V spectrometer (IONTOF GmbH, Münster, Germany) was used in this study. Compared to earlier works, ${ }^{1}$ optimized SIMS operation conditions were used. ${ }^{2}$ The detailed comparison was described in another publication. ${ }^{3}$ A $25 \mathrm{keV} \mathrm{Bi}_{3}{ }^{+}$cluster ion beam was the primary ion beam in this study. During measurements, the $\mathrm{Bi}_{3}{ }^{+}$beam was initially focused at around $200 \mathrm{~nm}$ diameter to obtain an observation

area of $2 \mu \mathrm{m}$ in diameter. ${ }^{2}$ In each depth profile experiment (Figure 2, region I), a wider pulse with a current of $1209 \mathrm{pA}$ and cycle time of $30 \mu \mathrm{s}$ was used initially before punching through the $100 \mathrm{~nm}$ SiN membrane. The punch through time was approximately $360 \mathrm{~s}$. After that the SiN membrane punchthrough, a wider pulse was used for another $150 \mathrm{~s}$ to obtain spectra with relatively better spatial resolution for image analysis (Figure 2, region II). In the end, a narrower pulse width was used for another $200 \mathrm{~s}$ to obtain data with better mass resolution (Figure 2, region III).

Dry biofilm and dry MM1 sample preparation

The biofilm was harvested in a tubular biofilm reactor. Each tubular biofilm reactor was made by the peroxide-cured silicone tubing (Masterflex, length $300 \mathrm{~mm}$, internal diameter $3.2 \mathrm{~mm}$ ). In the beginning, each reactor was inoculated using diluted overnight cultures in MM1 medium (OD $600 \sim 0.1)$. After 2 hrs. stop-flow for cells' initial attachment, MM1 medium was continuously supplied with a flow rate of 5.4 $\mathrm{ml} / \mathrm{hrs}$. for biofilm growth. After $168 \mathrm{hrs}$., the biofilms (wet biofilms) formed inside the tubular biofilm reactors were harvested and extracted onto a glass slide. Then the wet biofilms were further dried by air for $120 \mathrm{hrs}$. After totally dried, the dry biofilm samples were sent for ToF-SIMS dry sample analysis. If the samples had too much salt content, it would affect ToF-SIMS measurements due to the matrix effects. In this case, the wet samples need to be washed by DI water three times before applying the air-drying process.

The dry MM1 sample was made by dispensing a drop of liquid MM1 medium solution onto a clean glass slide or silicon wafer, followed by the freeze-drying process $\left(-80^{\circ} \mathrm{C}\right)$ for $120 \mathrm{hrs}$. 


\section{Supporting Information}

\section{Supplemental Figures}

Figure S1 provides more information conerning how the correlative imaging between SIM and SIMS was conducted.

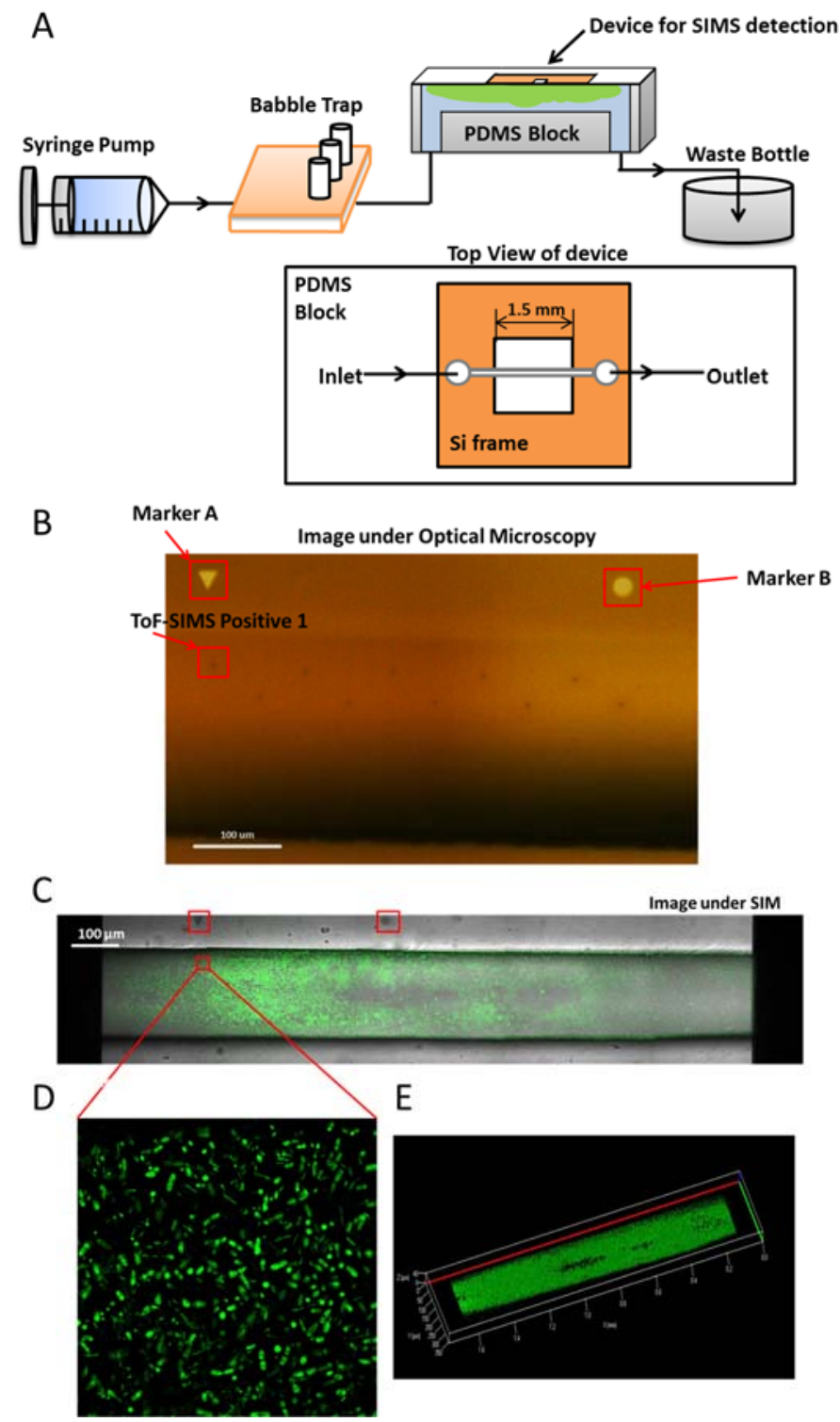

Figure S1. (A) Schematic illustration of the experimental setup in this study. Device fabrication was described in our an previous study. ${ }^{4}$ Briefly, soft photolithography was used in making the microfluidic channel on the polydimethylsiloxane (PDMS) block. The size of microfluidic channel was $1.5 \mathrm{~mm}$ (length) $\times 200 \mu \mathrm{m}$ (width) $\times 500 \mu \mathrm{m}$ (depth). Then two holes were punched at the two ends of the microfluidic channel for (1) bacterial inoculation and (2) medium supply. (B)The five positive points and five negative points inside SALVI channel under optical microscope, the three red squares are positive point 1, triangle shape and round shape; (C) The same SALVI channel under structured illumination microscopy (SIM) (using 10× lens), the three red squares are point of positive point 1 , triangle shape and round shape; (D) The SIM image (using 100× oil-immersion lens) on the red square of the point of positive point 1; (E) The SIM 3D image (using 10× lens) of the biofilm in the SALVI microfluidic channel. 


\section{Supporting Information}

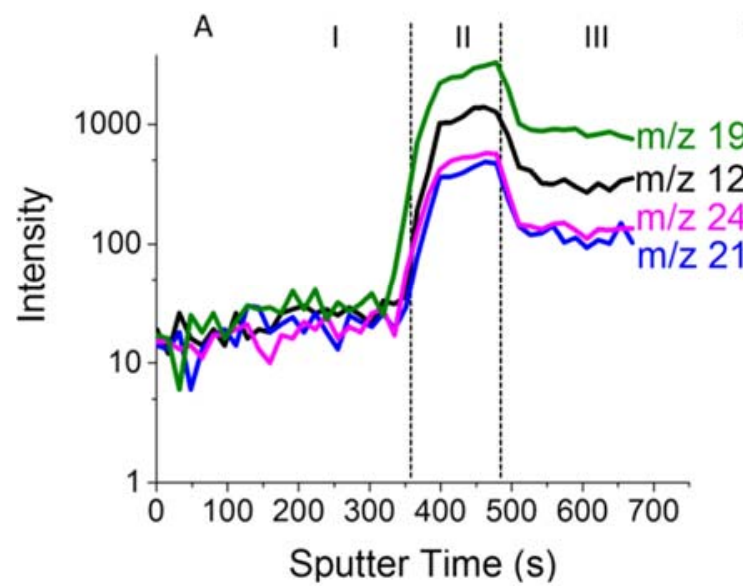

B
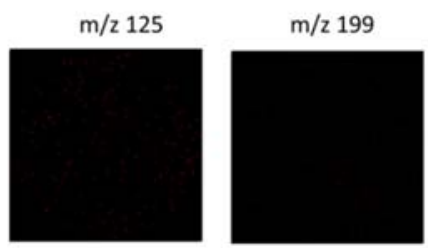

$\mathrm{m} / \mathrm{z} 211$

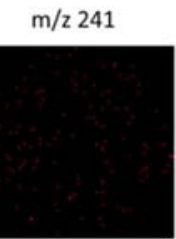

II

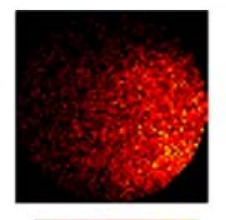

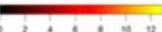

$\left(\mathrm{H}_{2} \mathrm{O}\right)_{6} \mathrm{OH}^{-}$

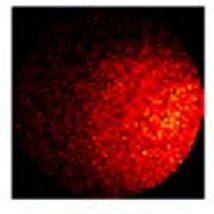

bin n

Lauric acid
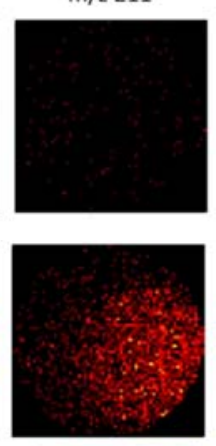

$\ldots, \ldots$

$\mathrm{C}_{2} \mathrm{H}_{3}\left(\mathrm{CH}_{2}\right)_{10} \mathrm{COO}$ -

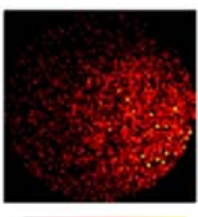

Riboflavin

Figure S2 provides depth profile of the untreated biofilm and reconstructed represented 2D images from region II in the depth profile time series in the negative mode. A) Depth profiling of the untreated biofilm and (B) representative 2D images (image size: $2 \mu \mathrm{m} \times 2 \mu \mathrm{m}$ ) reconstructed from region II in the negative depth profile. The ion doses are estimated as $1.432 * 10^{16}$ ions $/ \mathrm{cm}^{2}$. Pixel size is $31.25 \mathrm{~nm}$. 


\section{Supporting Information}

In order to ascertain possible peak identifications, we conducted SIMS analyses of a series of reference samples. In the following, we present the comparison results. Dry sample preparation was detailed in the SI experimental section. Liquid samples were analyzed using the SALVI approach. First, we show the comparison between dry and liquid S. oneidensis biofilm in Figures S3A and S3B. Second, we show the comparison between dry and liquid biofilm medium in Figures S3C and S3D. Third, we show comparison of in situ liquid live biofilm and dry reference samples in Figures S4A and S4B. The reference samples include N-Butyryl-L-Homoserine lactone, 2-HEPtyl-3-Hydroxy-4(IH)-Quinolone, Palmitic acid, and Riboflavin.
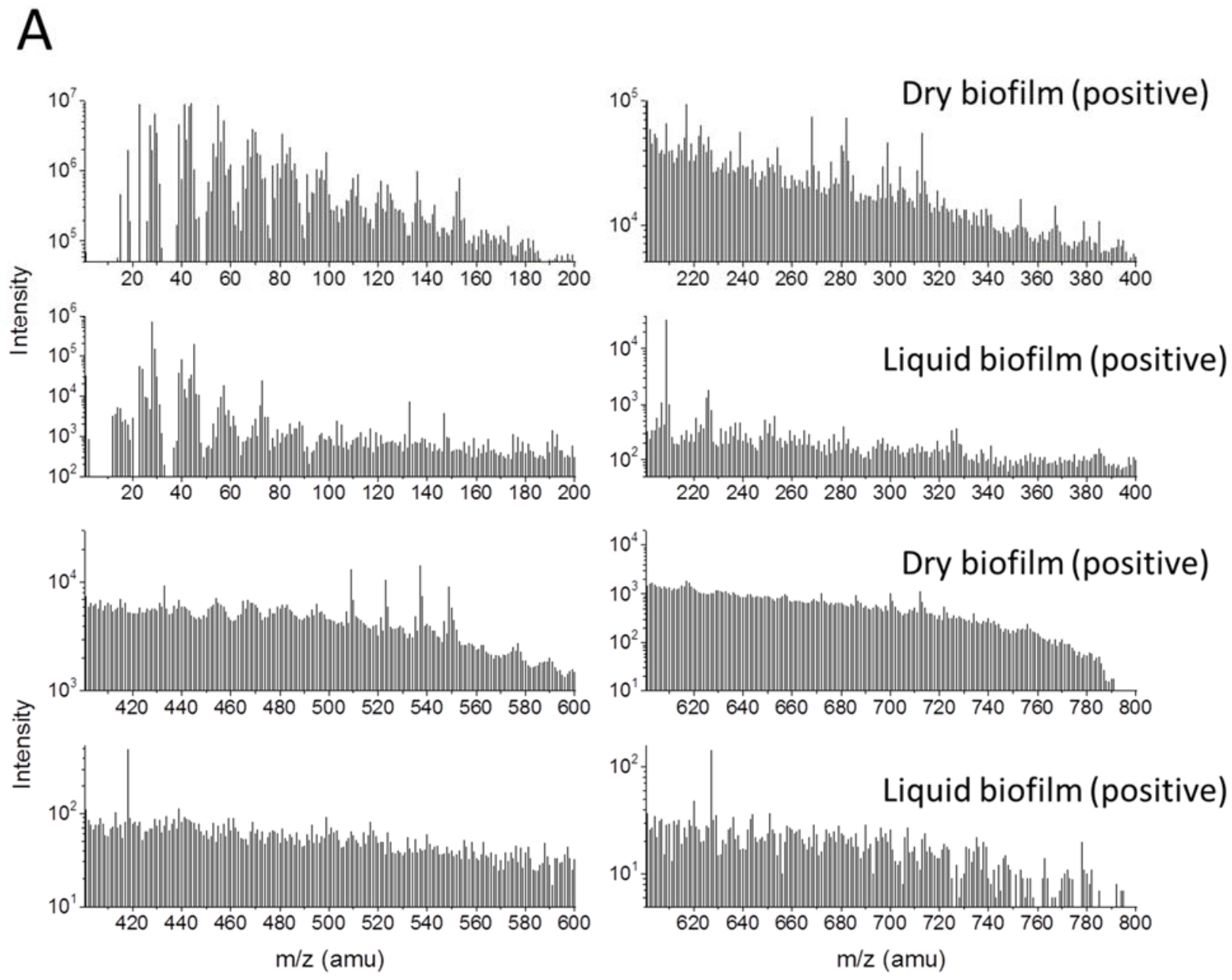

Figure S3A. Comparison of biofilm dry sample and liquid sample SIMS m/z spectral data (m/z $\left./ \mathrm{z}^{+} 0-800\right)$ in the positive mode 


\section{Supporting Information}

B
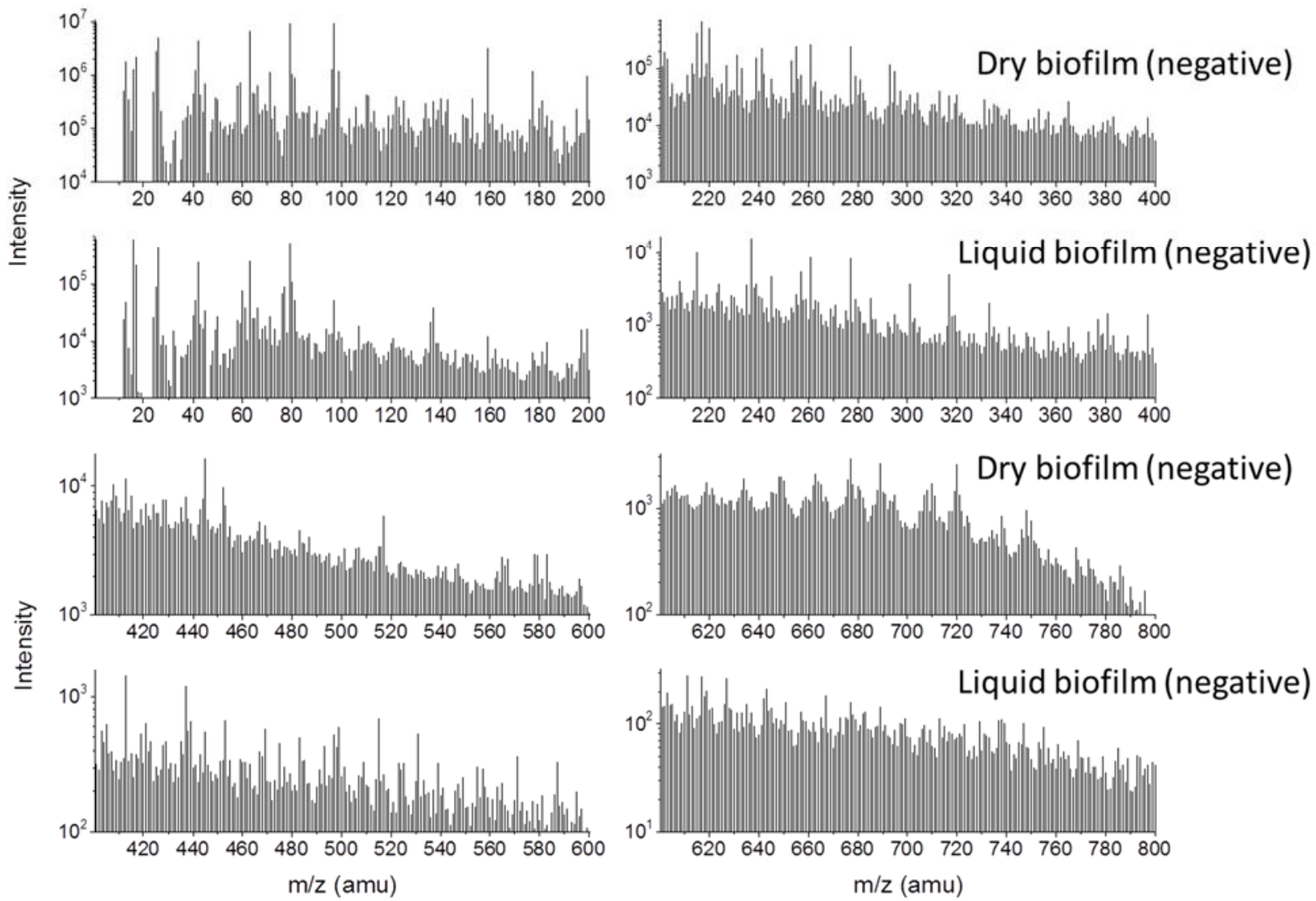

Figure S3B. Comparison of SIMS m/z spectra of biofilm dry sample and liquid sample $\left(\mathrm{m} / \mathrm{z}^{-} 0-800\right)$ in the negative mode 


\section{Supporting Information}

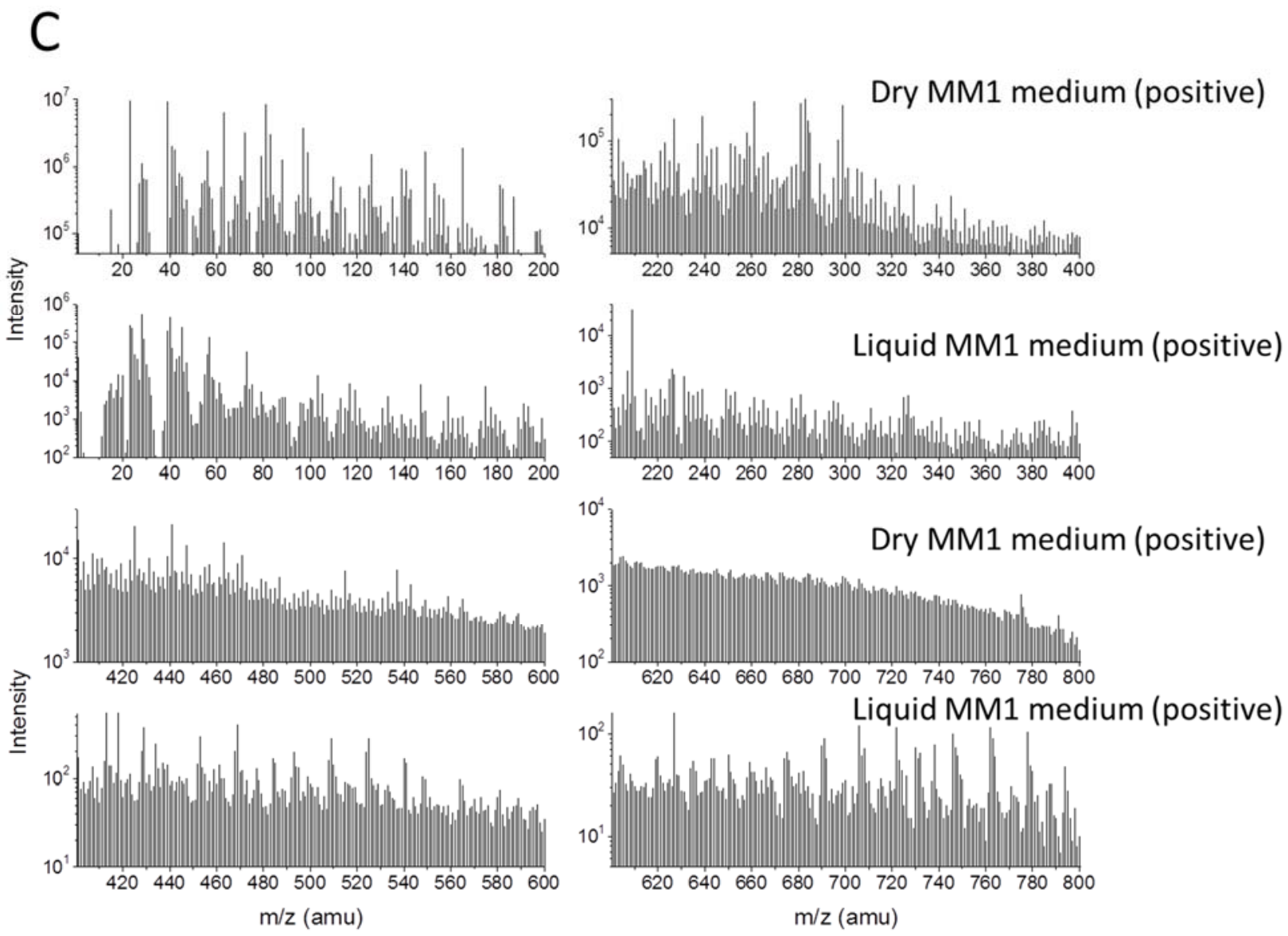

Figure S3C. Comparison of SIMS m/z spectra of MM1 medium dry sample and liquid sample $\left(\mathrm{m} / \mathrm{z}^{+} 0\right.$ $800)$ in the positive mode. 


\section{Supporting Information}

D

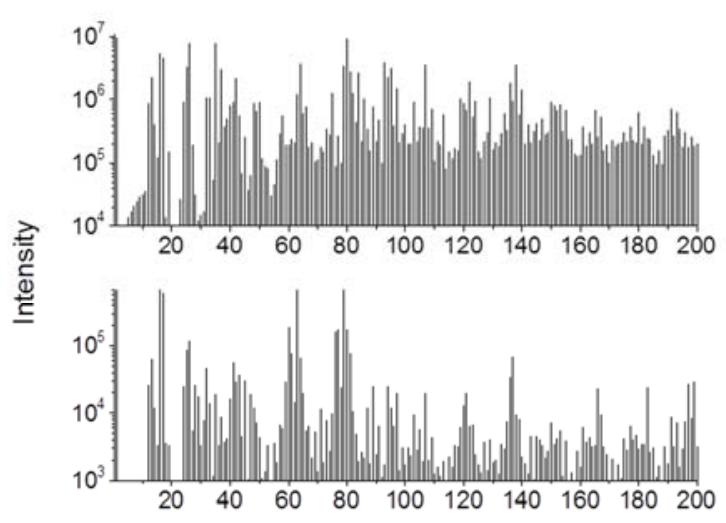

Dry MM1 medium (negative)
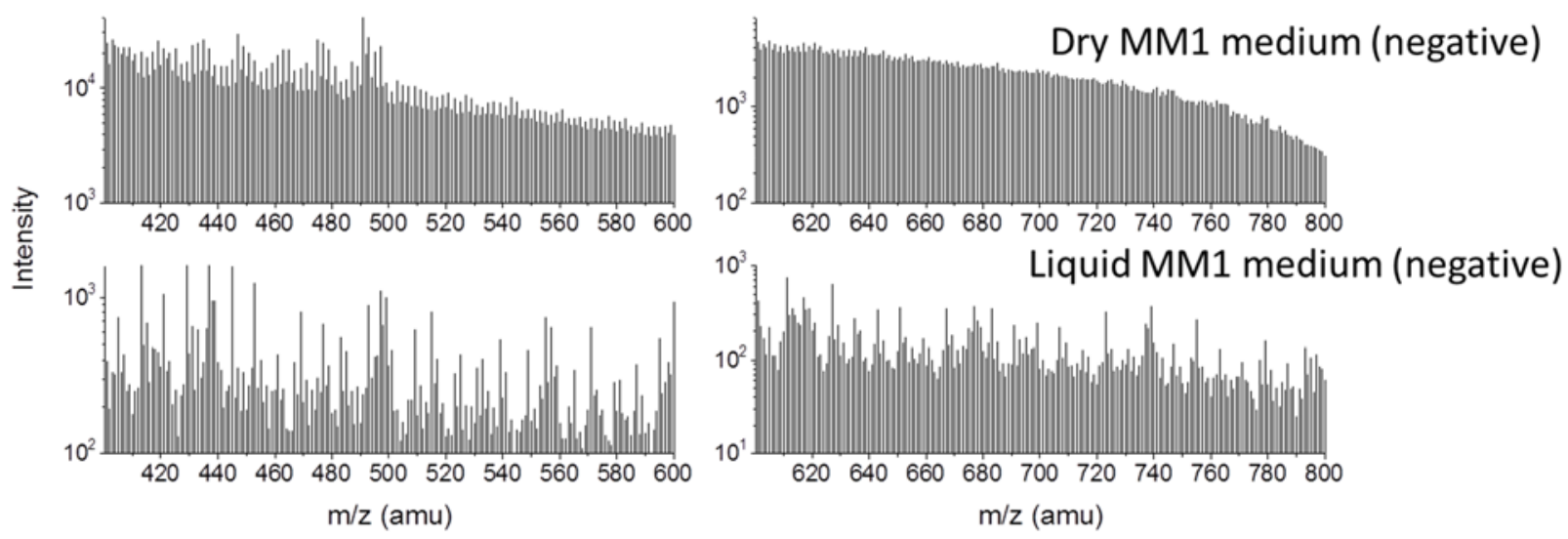

Figure S3D. Comparison of SIMS m/z spectra of MM1 medium dry sample and liquid sample (m/z $\mathrm{z}^{-}$$800)$ in the negative mode. 


\section{Supporting Information}
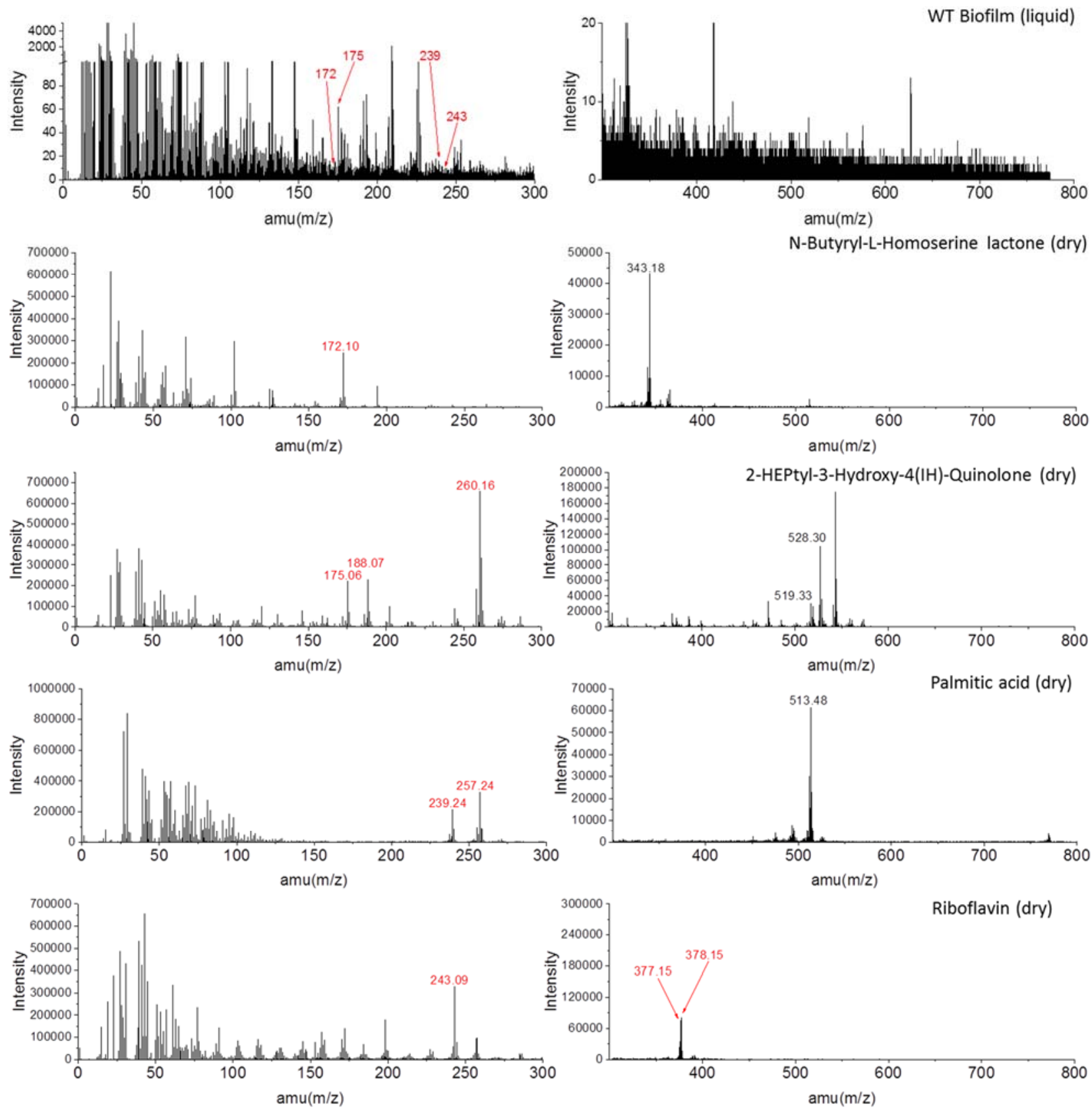

Figure S4A. Comparison of SIMS m/z spectra of in situ liquid live wild type (WT) biofilm and dry reference samples in the positive mode. From top to bottom: in situ live wild type (WT) biofilm (liquid sample); N-Butyryl-L-Homoserine lactone (dry sample control); 2-HEPtyl-3-Hydroxy-4(IH)-Quinolone (dry sample control); Palmitic acid (dry sample control); Riboflavin (dry sample control). 


\section{Supporting Information}
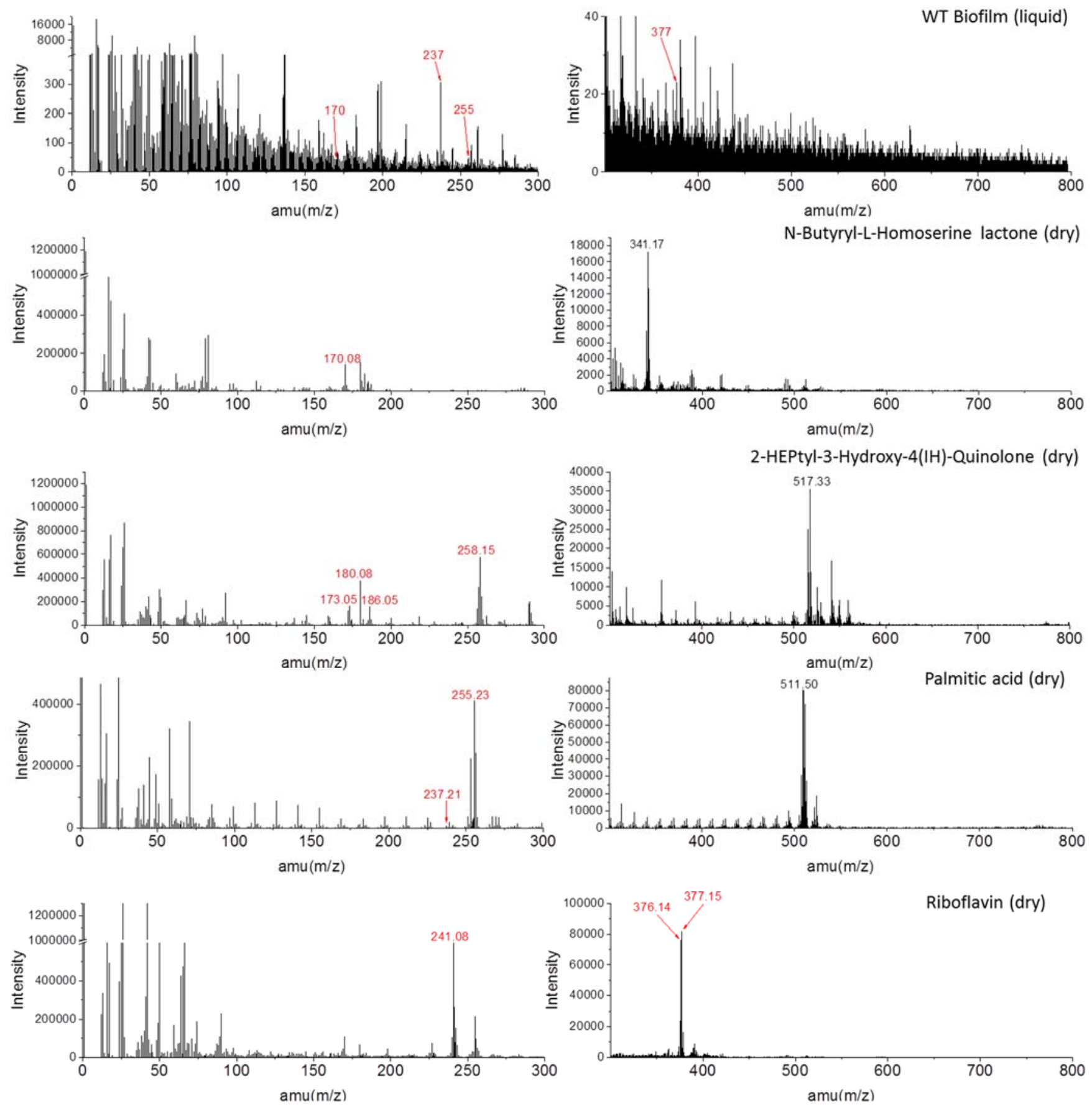

Figure S4B. Comparison of SIMS m/z spectra of in situ liquid live wild type (WT) biofilm and dry reference samples in the negative mode. From top to bottom: in situ live wild type (WT) biofilm (liquid sample); N-Butyryl-L-Homoserine lactone (dry sample control); 2-HEPtyl-3-Hydroxy-4(IH)-Quinolone (dry sample control); Palmitic acid (dry sample control); Riboflavin (dry sample control). 


\section{Supporting Information}

Figure S5 provides the $\mathrm{m} / \mathrm{z}$ spectra of all samples in the positive mode in the $\mathrm{m} / \mathrm{z}^{+}$range of $1-500$ (A); and 501-800 (B).

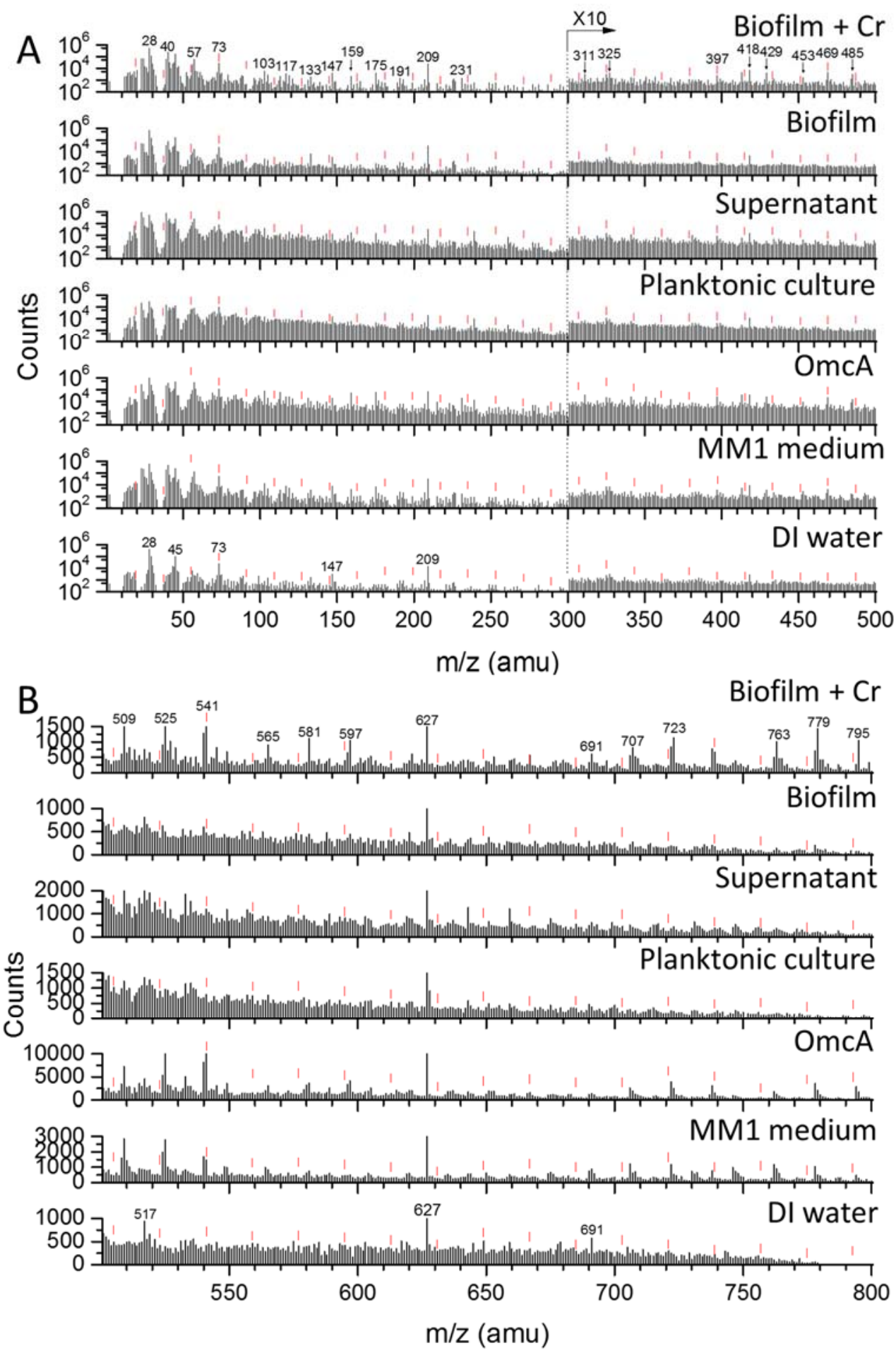

Figure S5. Spectra of all samples from $\mathrm{m} / \mathrm{z}^{+} 1$ to 500 (A) and 501 to 800 (B) in the positive mode. Red bars depict the location of water cluster peaks. 


\section{Supporting Information}

Figure S6 shows additional spectra data of all samples in the $\mathrm{m} / \mathrm{z}^{-}$range of 1-500 (A) and 501-800 (B) in the negative respectively.

A

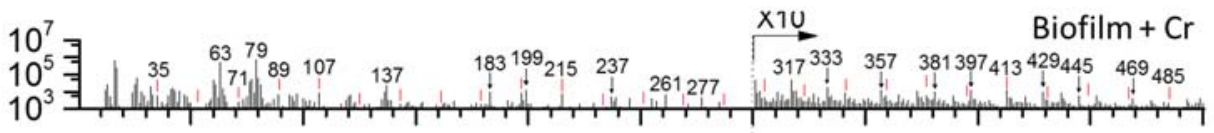

$$
10^{7}
$$
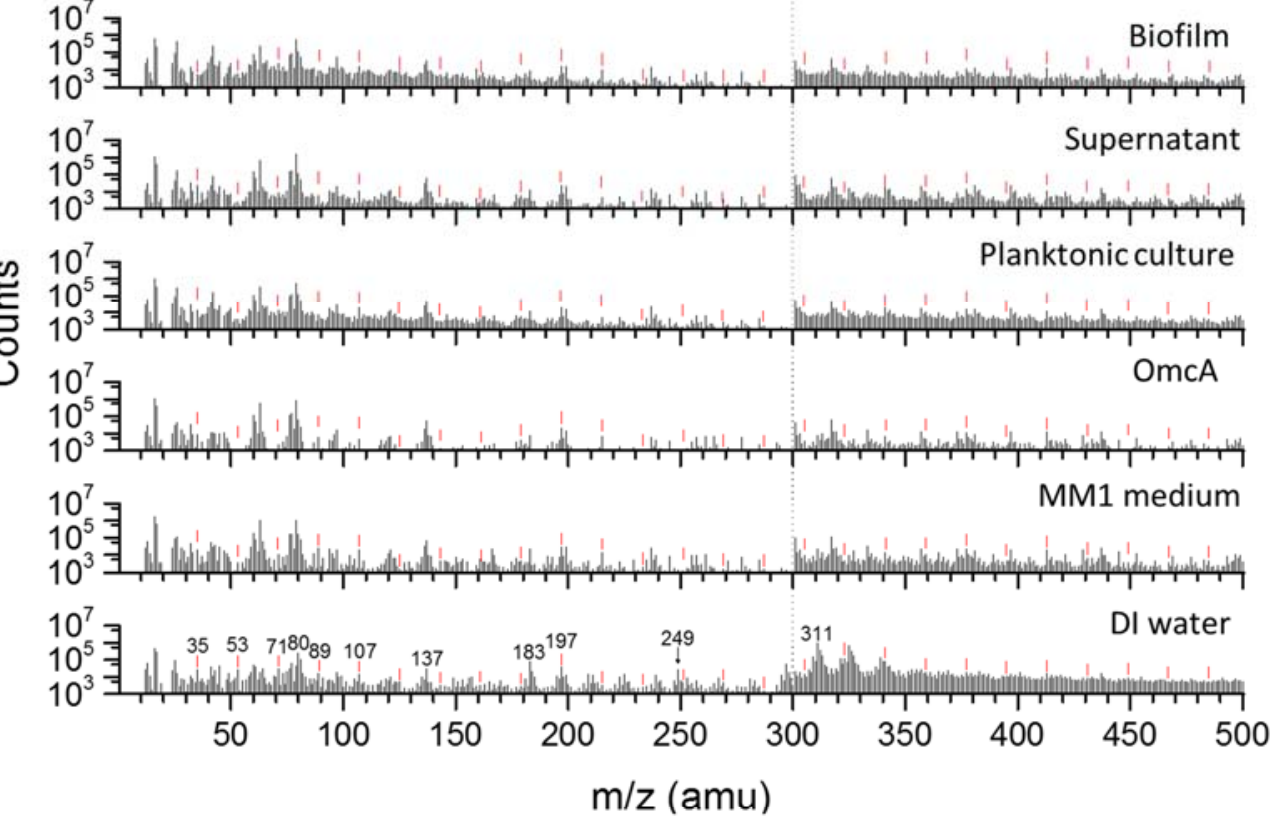

B
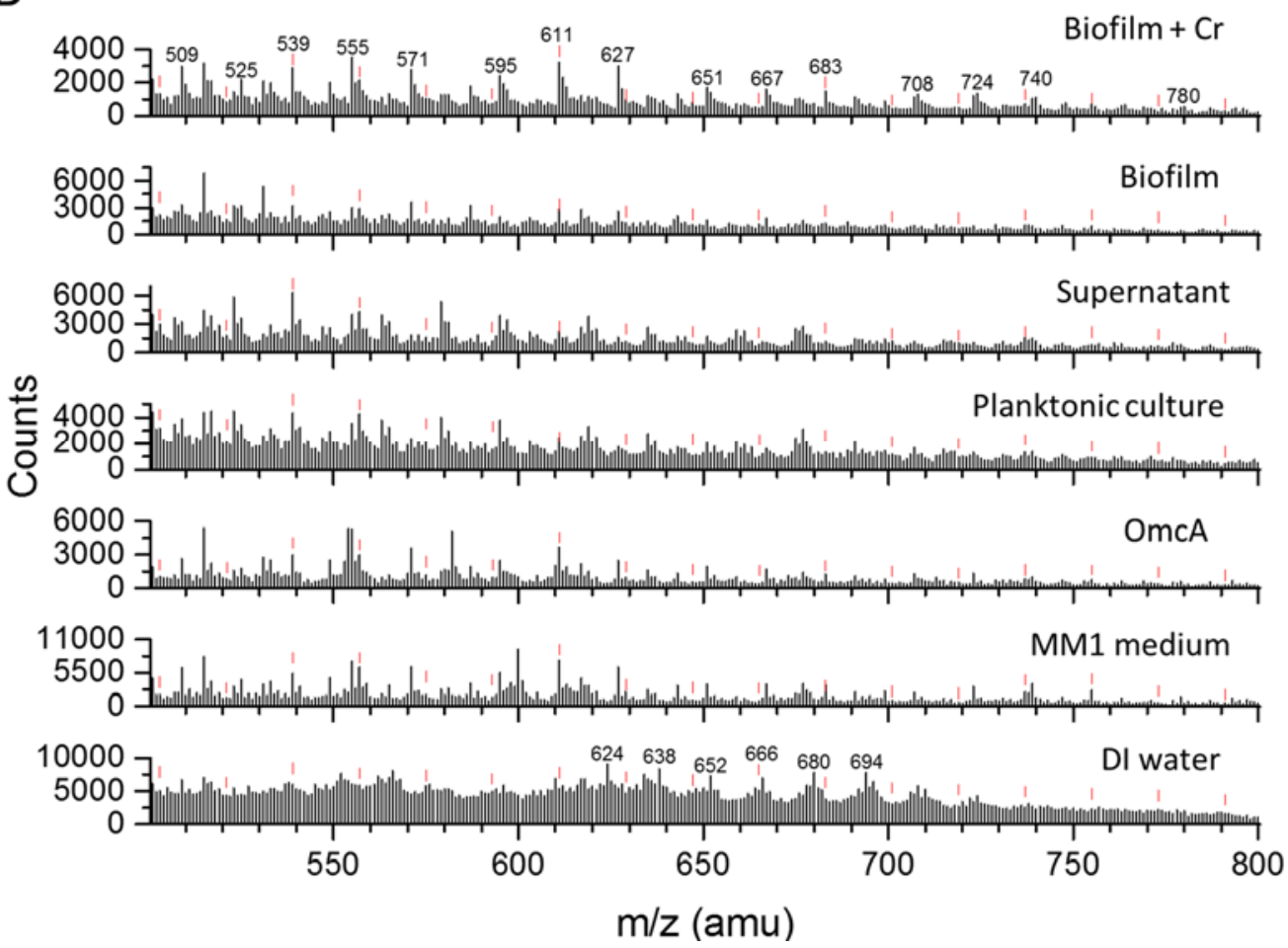

Figure S6. Negative spectra of all samples in the $\mathrm{m} / \mathrm{z}^{-}$range of 1-500 (A) and 501-800 (B) Red bars depict the location of water cluster peaks. 


\section{Supporting Information}

Figure S7 shows the SIMS analysis of untreated biofilms at multiple locations along the microchannel in the positive mode.
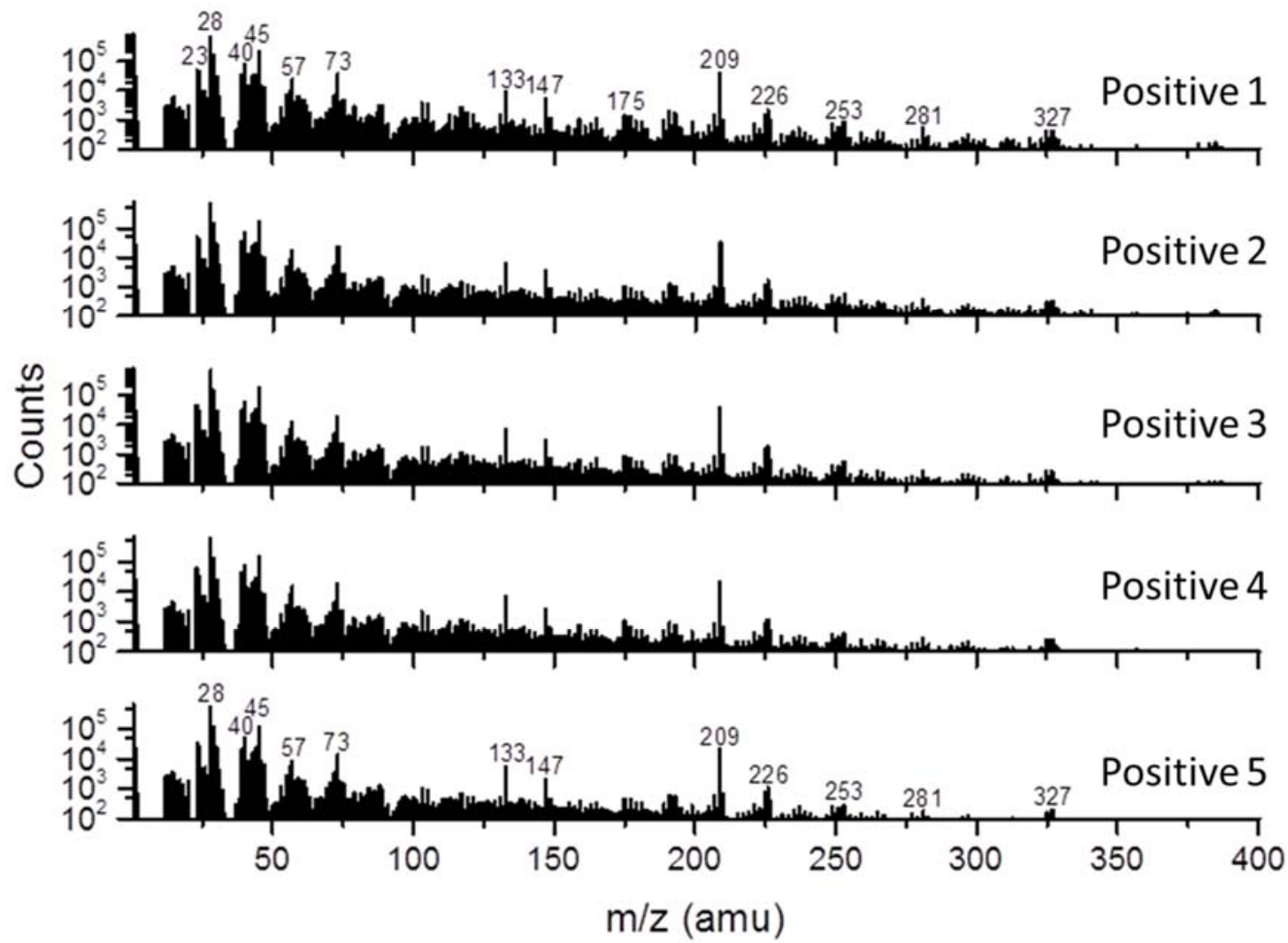

Figure S7. Comparison of the untreated biofilm analysis at different locations in the positive mode. 


\section{Supporting Information}

Figure S8 shows the negative spectra data of the untreated biofilm analysis at multiple locations along the microfluidic channel where biofilms were cultivated.
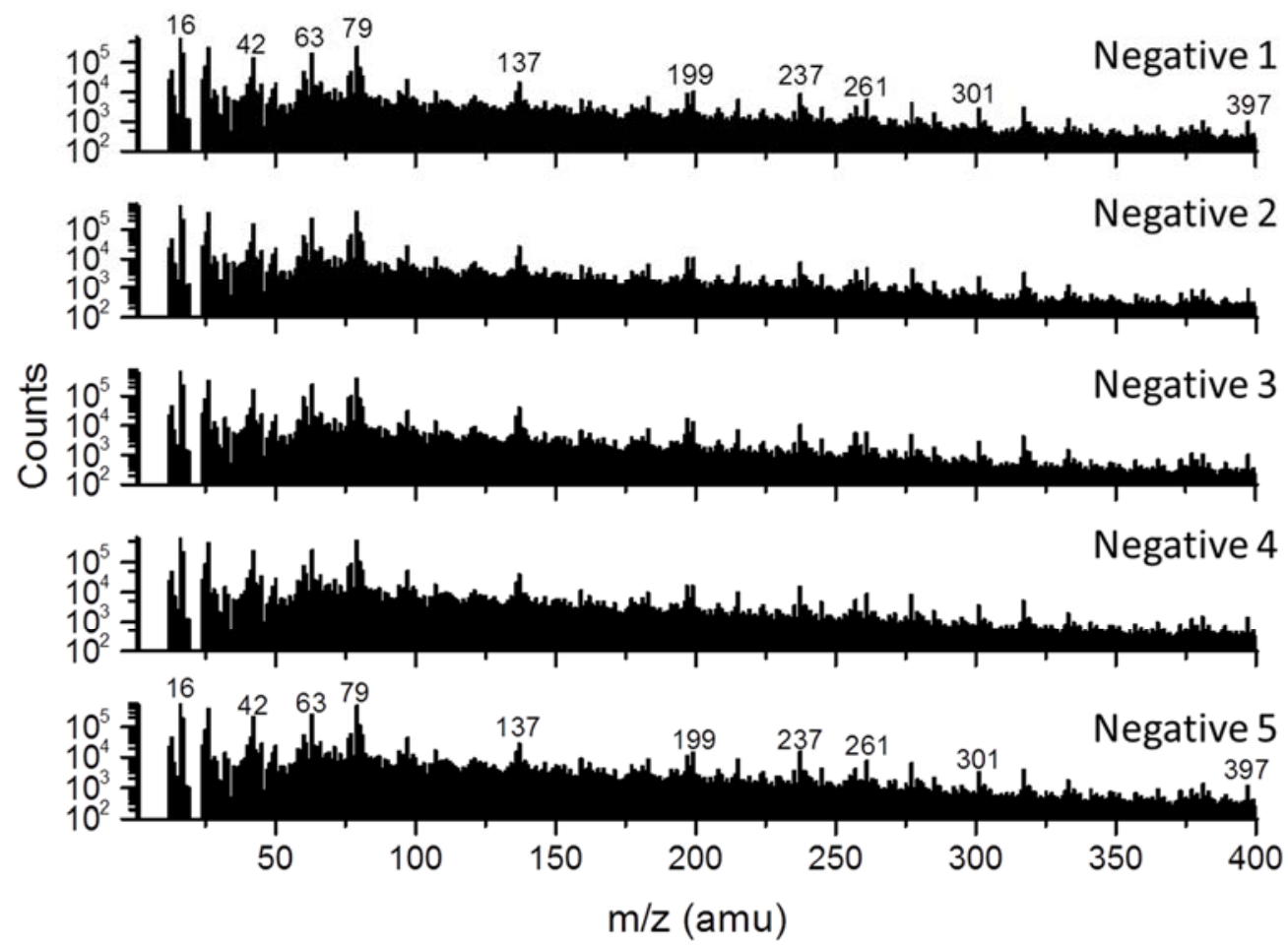

Figure S8. Comparison of the untreated biofilm analysis at different locations in the negative mode. 


\section{Supporting Information}

Figure S9 shows comparison of characteristic fragments among different samples.
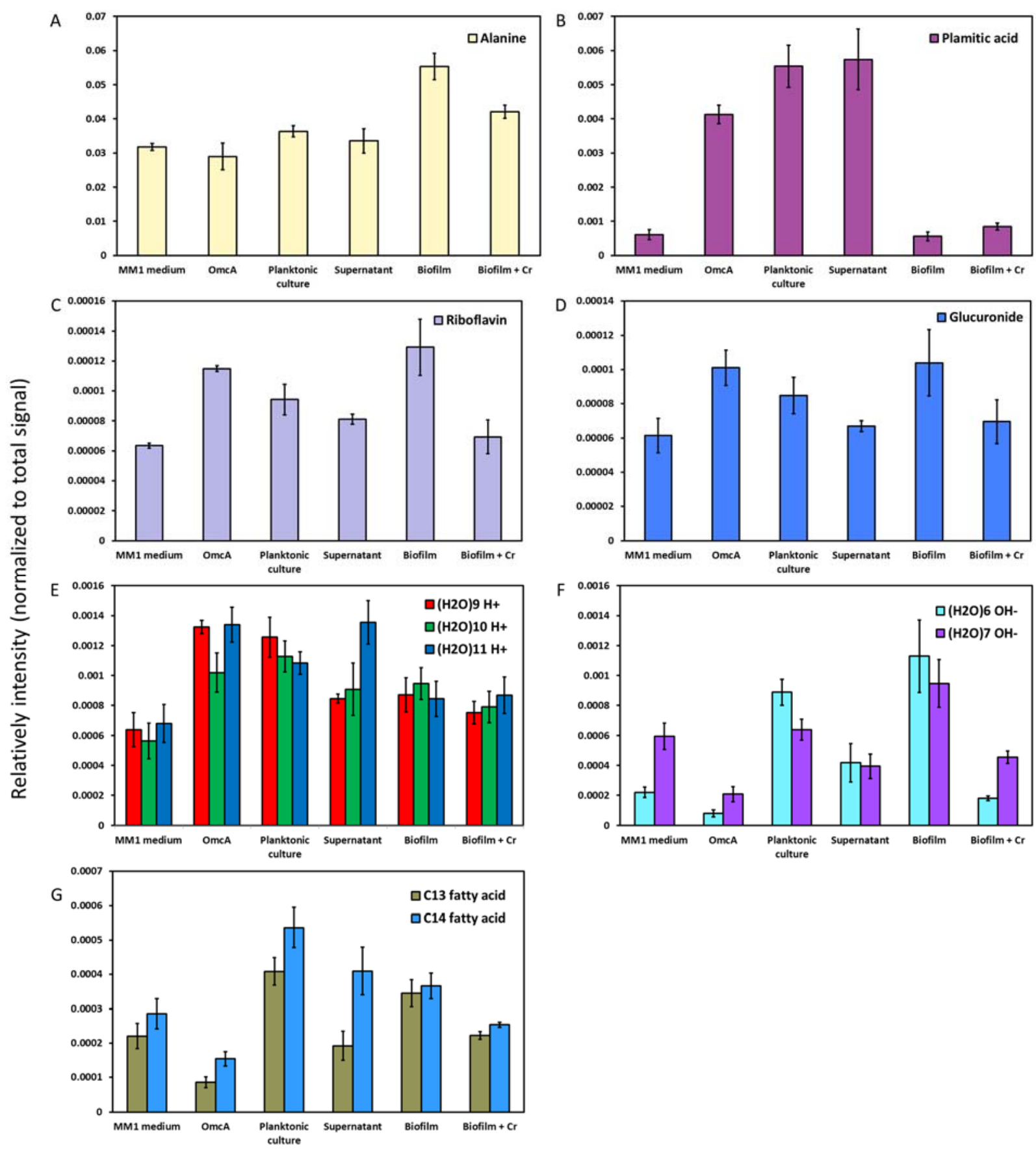

Figure S9. The bar plot for normalized peak intensities in the positive ion mode (A) alanine (B) palmitic acid (one of the most common fatty acid) (C) riboflavin (D) glucuronide (E) water cluster, and in negative ion mode $(\mathrm{F})$ water cluster $(\mathrm{G})$ fatty acid among different experimental groups. Data are normalized to the total ion intensity. A-E are data in the positive ion mode, and F, $\mathrm{G}$ are the negative ion mode. Values are means \pm standard deviations $(n \geq 3)$. 


\section{Supporting Information}

Spectral PCA was performed. Selected peaks in the $\mathrm{m} / \mathrm{z}^{+}$range 1 to 800 atomic mass unit (amu) were used in this analysis. After normalization to selective peaks' total ion intensity, the PCA score plot with first two principal components (PCs) was depicted in Figure 3A. , PC1 explained 52.98\% of the variance and PC2 explained 35.51\% of the variance. The peaks selected for spectral PCA were based on following criteria: 1) The peak intensity was more than $0.5 \%$ of the total ion counts; 2 ) The peak intensity was 3 times higher than its neighborhood; and 3 ) The peak representing important EPS chemical components based on previous bulk, dry sample, or wet sample analysis. ${ }^{8-11}$ The interference peaks from PDMS and primary ion beam are excluded from peaks selection.

Figure S10 provides additional score plots and loading plots for the positive spectral PCA using selected peaks. The selected peak list in the positive mode is summarized in Tables S4A and S4B.

Figure S11 provides additional score plots and loading plots for the negative spectral PCA using selected peaks. The selected peak list in the negative mode is summarized in Tables S5A and S5B.

Figure S12 shows spectral PCA results using all peaks in the positive mode. These analysis results essentially are in agreement with the selected peaks spectral PCA. This finding indicates that the selected peaks used in the PCA are representative of the group of samples. Moreover, they accentuate the essence of the chemistry of the biofilm and its response to chromium (VI) heavy metal exposure.

Similarly, Figure S13 shows PCA results using all peaks in the negative mode. These analysis results essentially are in agreement with the selected peaks PCA. This finding indicates that the selected peaks used in the PCA are representative of the group of samples. Moreover, they accentuate the essence of the chemistry of the biofilm and its response to chromium (VI) heavy metal exposure. 


\section{Supporting Information}
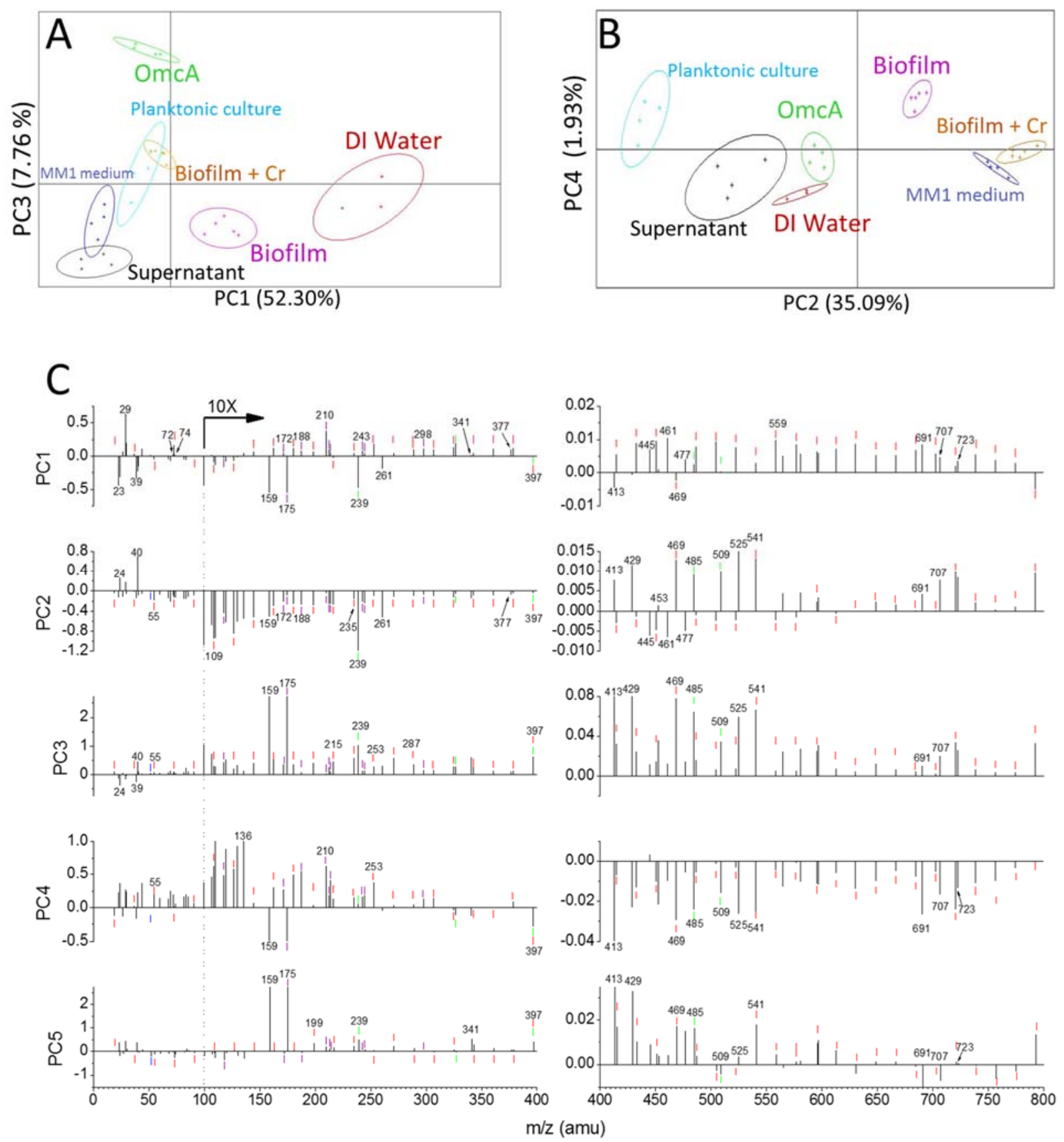

Figure S10. The score plot of PC1 vs. PC3 (A) and PC1 vs. PC4 (B) in the positive selected peaks spectral PCA. The loading plots of each PC are depicted in (C). Red bars indicate the location of water cluster peaks, purple quorum sensing signal peaks, and green fatty acid peaks. 
Supporting Information

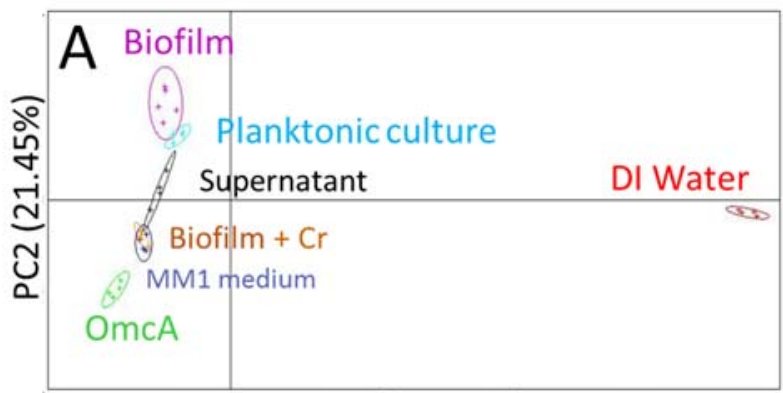

PC1 (63.52\%)

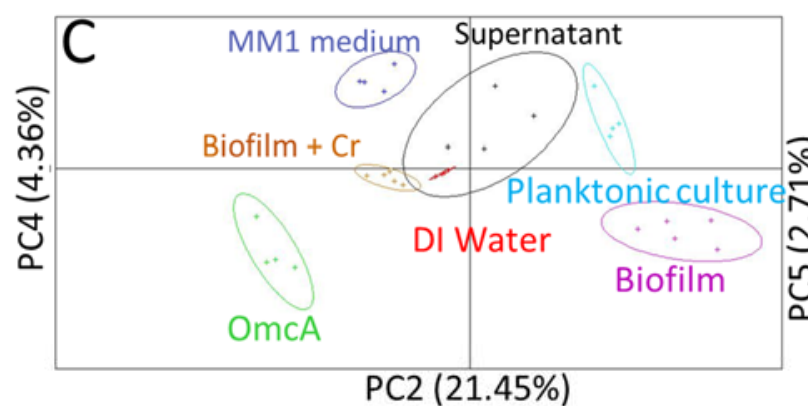

PC2 (21.45\%)
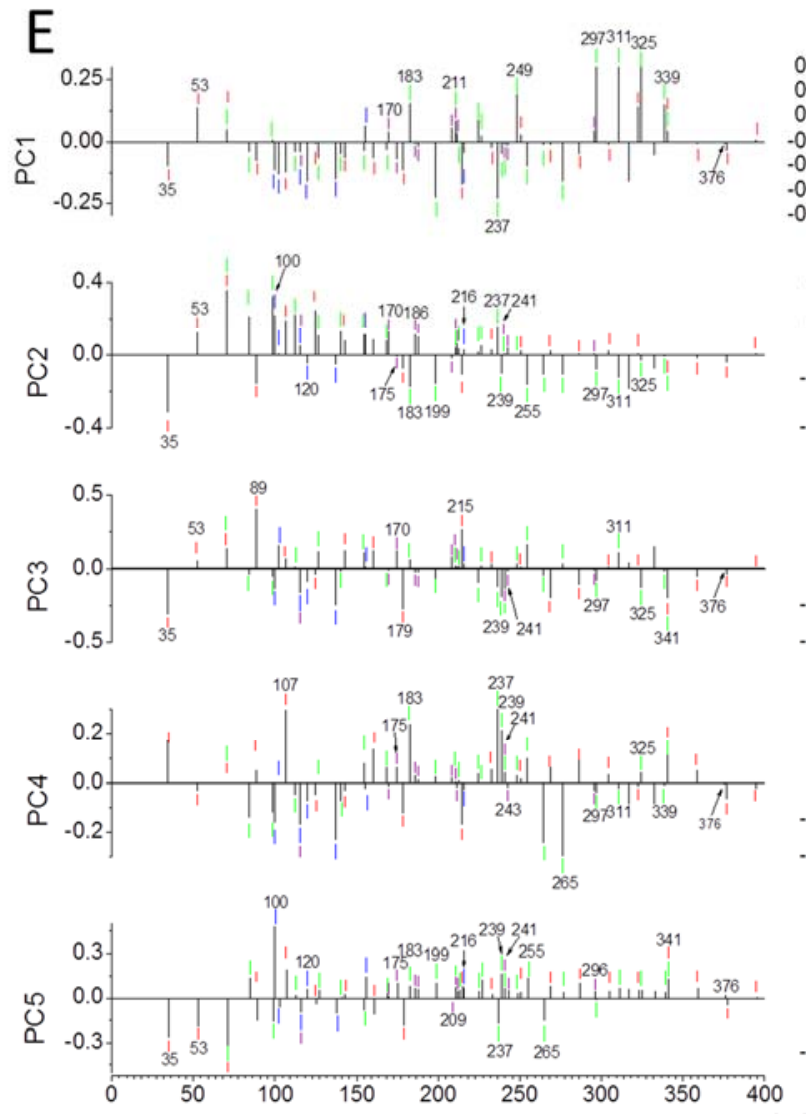
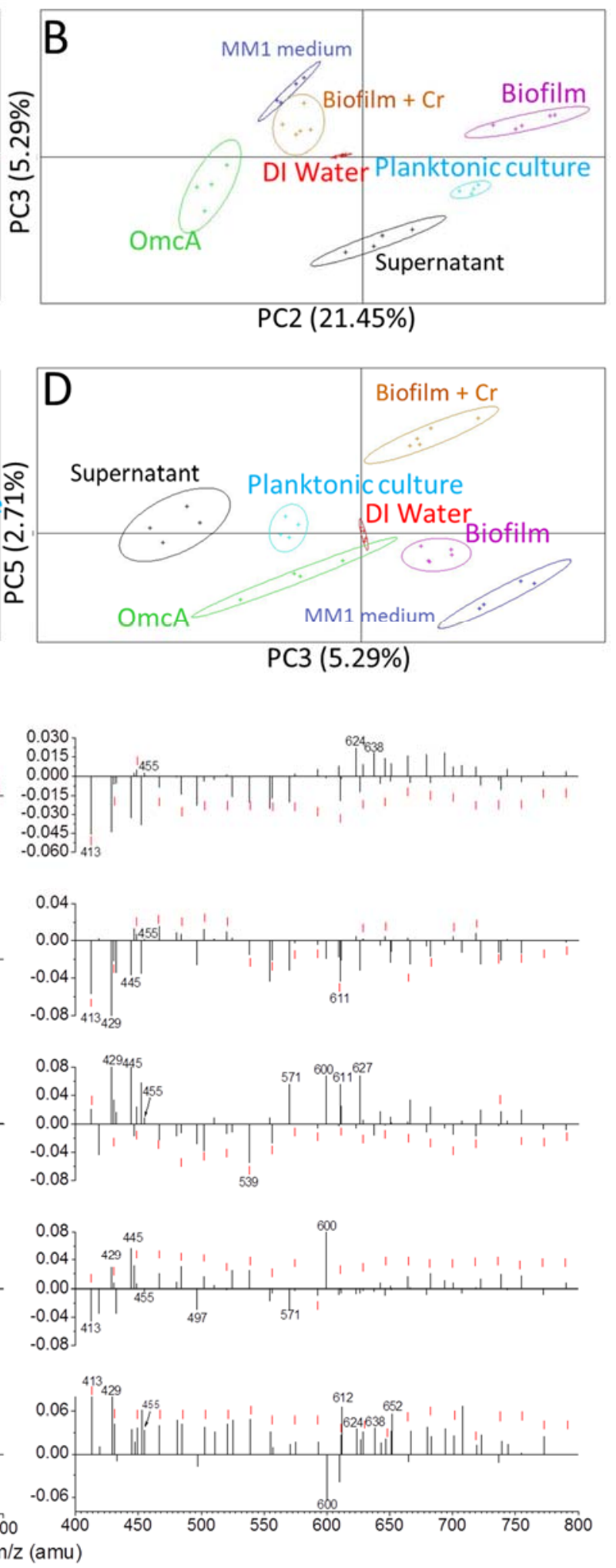

Figure S11. The score plots of PC1 vs. PC2 (A), PC1 vs. PC3 (B), PC1-PC4 (C), and PC3-PC5 (D) in the negative selected peaks spectral PCA. The loading plots of each PC are depicted in E. Red bars indicate the location of water cluster peaks, blue chromium reduction related peaks, purple quorum sensing signal peaks, and green fatty acid peaks. 
Supporting Information
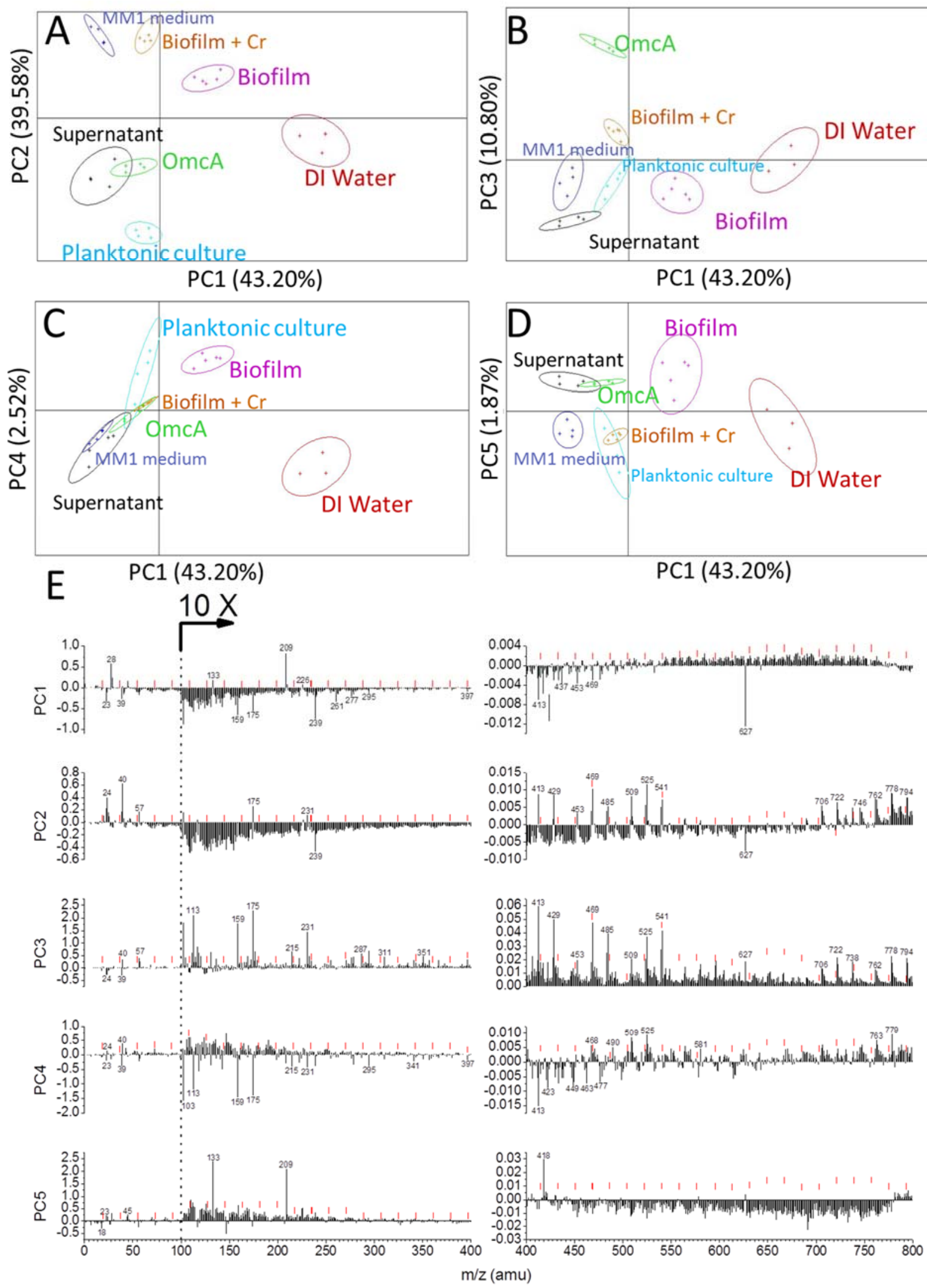

Figure S12. Positive spectral PCA of all peaks. (A) Score plots and (B) loading plots of each PC. Red bars indicate the location of water cluster peaks. 


\section{Supporting Information}

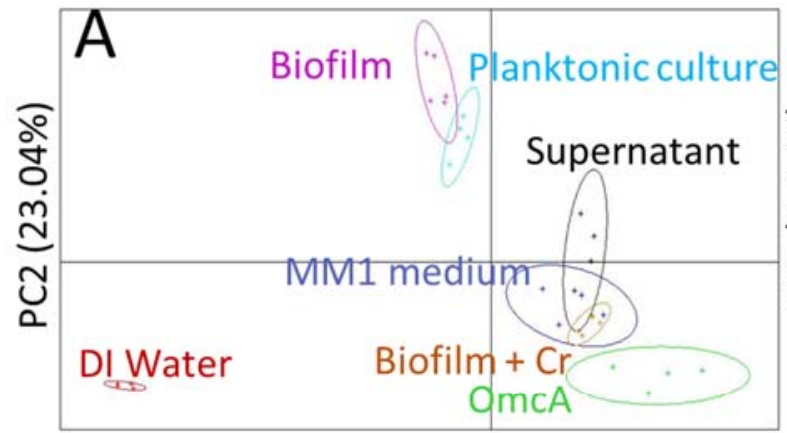

PC1 (66.22\%)

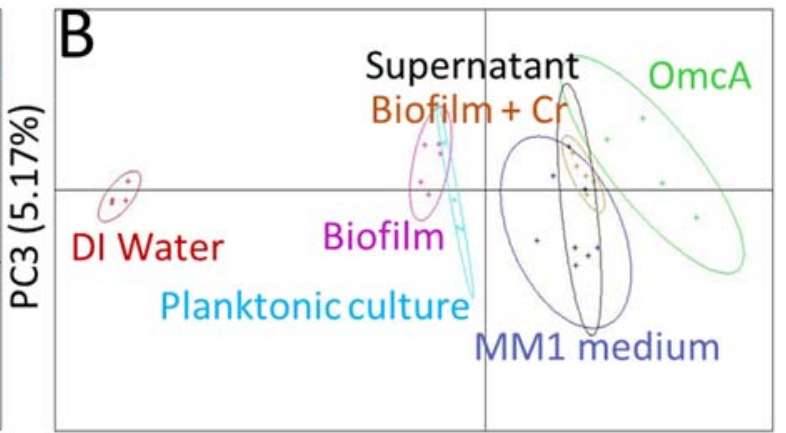

PC1 (66.22\%)
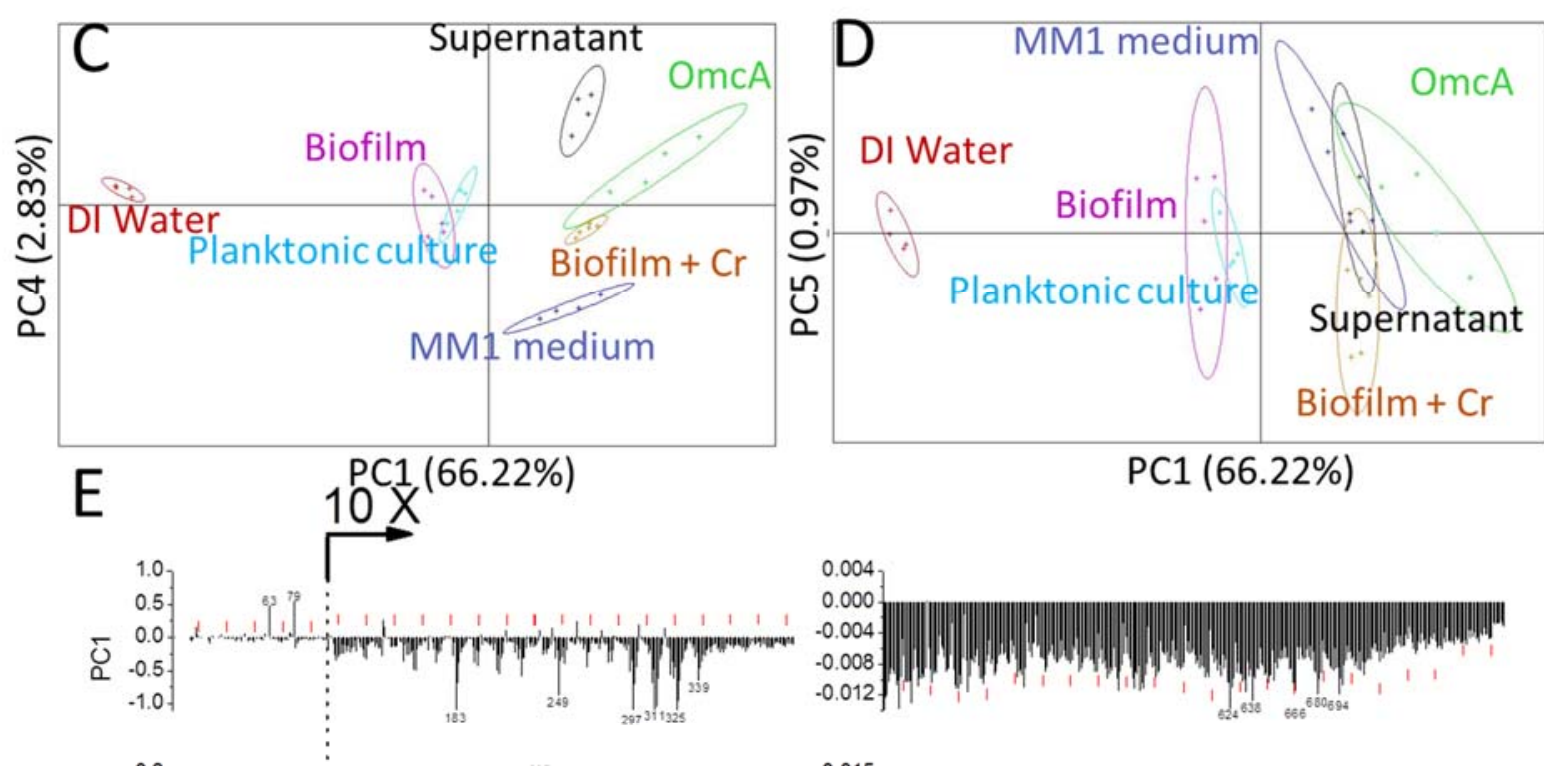

PC1 (66.22\%)
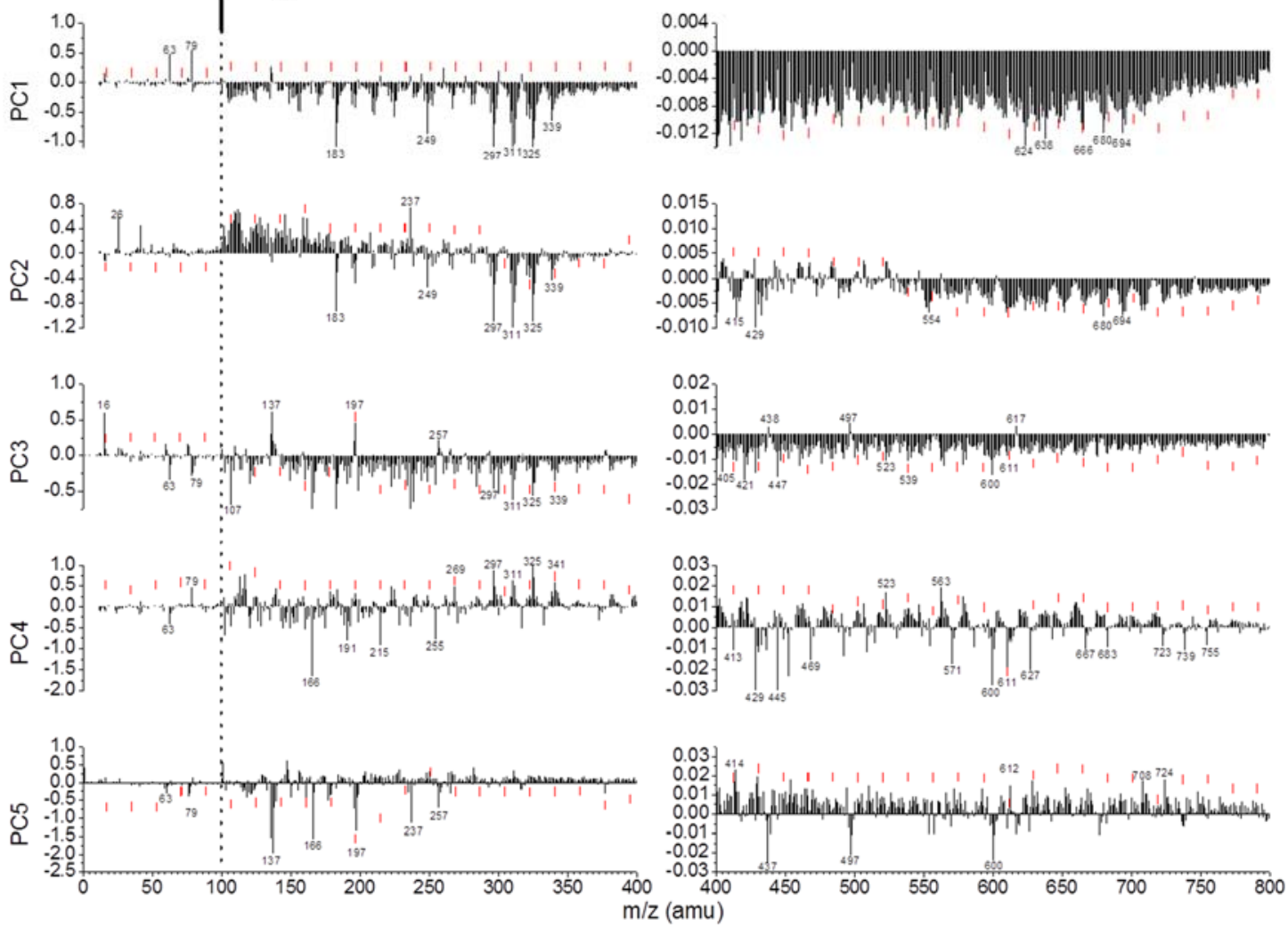

Figure S13. Negative spectral PCA of all peaks. (A) Score plots and (B) loading plots of each PC. Red bars indicate the location of water cluster peaks. 


\section{Supporting Information}

\section{Supplemental Tables}

Table S1. Summary of sample descriptions.

\begin{tabular}{|c|c|c|}
\hline Sample & Brief name & Chemical details/ Preparation process \\
\hline $\begin{array}{l}\text { Shewanella } \\
\text { oneidensis MR-1 } \\
\text { wild type (WT) } \\
\text { biofilm }+\mathrm{Cr}\end{array}$ & Biofilm $+\mathrm{Cr}$ & $\begin{array}{l}\text { Inoculate diluted overnight MR- } 1 \text { WT cultures into SALVI } \\
\text { system. Stop the flow } 4 \mathrm{hrs} \text {. for initial attachment. Supply MM1 } \\
\text { medium with a flow rate of } 2.88 \mathrm{ml} / \text { day for biofilm growth. At } \\
\text { Day } 7 \text {, add } 100 \mu \mathrm{M} \mathrm{K} \mathrm{K}_{2} \mathrm{Cr}_{2} \mathrm{O}_{7} \text { into MM1 and treat the biofilms. } \\
\text { Harvest at Day } 9\end{array}$ \\
\hline $\begin{array}{l}\text { S. oneidensis MR- } \\
1 \text { WT biofilm* }\end{array}$ & $\begin{array}{l}\text { Biofilm/Untreated } \\
\text { biofilm }\end{array}$ & $\begin{array}{l}\text { Inoculate diluted overnight MR-1 WT cultures into SALVI } \\
\text { system. Stop the flow } 4 \text { hrs. for initial attachment. Supply MM1 } \\
\text { medium with a flow rate of } 2.88 \mathrm{ml} / \text { day for biofilm growth. } \\
\text { Harvest at Day } 9 .\end{array}$ \\
\hline $\begin{array}{l}\text { S. oneidensis MR- } \\
1 \text { Supernatant* }\end{array}$ & Supernatant & $\begin{array}{l}\text { Centrifuge the planktonic cultures at } 5000 \mathrm{~g} \text { for } 10 \mathrm{mins} \text {. Filter } \\
\text { the supernatant by } 0.22 \mu \mathrm{m} \text { membrane filter unit. }\end{array}$ \\
\hline $\begin{array}{l}\text { S. oneidensis MR- } \\
1 \text { Planktonic } \\
\text { culture* }\end{array}$ & $\begin{array}{l}\text { Planktonic/Planktonic } \\
\text { culture }\end{array}$ & $\begin{array}{l}\text { Inoculate } 20 \mu \mathrm{l} \text { of overnight MR- } 1 \mathrm{WT} \text { cultures into } 2 \mathrm{ml} \text { of } \\
\text { MM1 medium. Grow in } 30 \text { degree, } 200 \mathrm{rpm} \text { for } 24 \mathrm{hrs} \text {. }\end{array}$ \\
\hline $\begin{array}{l}\text { OmcA protein } \\
\text { film }\end{array}$ & OmcA & $\begin{array}{l}\text { Load the } 100 \mathrm{ul} \text { OmcA samples into the reservoir of centrifugal } \\
\text { filter unit (Millipore); add buffer; Centrifuge the samples at } \\
4000 \mathrm{~g} \text { for } 20 \text { mins; Discard the flow through; } \\
\text { Add } 1 \mathrm{ml} \mathrm{MM} 1 \mathrm{medium} \text { into the reservoir of centrifugal filter } \\
\text { unit; Centrifuge the samples at } 4000 \mathrm{~g} \text { for } 20 \text { mins; repeat twice } \\
\text { Collect the samples in the reservoir for ToF-SIMS experiment, } \\
\text { and discard the flow through; }\end{array}$ \\
\hline $\begin{array}{l}\text { Chemically } \\
\text { defined modified } \\
\text { M1 medium }\end{array}$ & MM1 & $\begin{array}{l}\text { Contain } 30.00 \mathrm{mM} \text { HEPES, } 7.50 \mathrm{mM} \mathrm{NaOH}, 28.04 \mathrm{mM} \\
\mathrm{NH} 4 \mathrm{Cl}, 1.34 \mathrm{mM} \mathrm{KCl}, 4.35 \mathrm{mM} \mathrm{NaH} \mathrm{PO}_{4}, 0.68 \mathrm{Mm} \mathrm{CaCl}_{2} \\
\text { supplemented with trace amounts of minerals, vitamins, and } \\
\text { amino acids }\end{array}$ \\
\hline DI water & DI water & $\begin{array}{l}\text { The DI water from pure water system in Pacific Northwest } \\
\text { National Laboratory's Biological Science Facility (BSF) }\end{array}$ \\
\hline
\end{tabular}

*: Shewanella is abbreviated as $S$. 


\section{Supporting Information}

Table S2A. Dry biofilm sample mass to charge ratios in the positive ion mode.

\begin{tabular}{|c|c|c|c|}
\hline Unit mass & Observed $\mathrm{m} / \mathrm{z}$ & Theoretical $\mathrm{m} / \mathrm{z}$ & Possible assignment \\
\hline 23 & 22.99 & 22.9892 & $\mathrm{Na}^{+}$ \\
\hline 28 & 27.98 & 27.9764 & $\mathrm{Si}^{+}$ \\
\hline 28 & 28.03 & 28.0313 & $\mathrm{C}_{2} \mathrm{H}_{4}^{+}$ \\
\hline 39 & 38.97 & 38.9632 & $\mathrm{~K}^{+}$ \\
\hline 40 & 39.96 & 39.962 & $\mathrm{Ca}^{+}$ \\
\hline 45 & 45.03 & 45.0335 & $\mathrm{C}_{2} \mathrm{H}_{5} \mathrm{O}^{+}$ \\
\hline 45 & 44.98 & 44.9793 & $\mathrm{SiOH}^{+}$ \\
\hline 57 & 57.03 & 57.034 & $\mathrm{C}_{3} \mathrm{H}_{5} \mathrm{O}^{+}$ \\
\hline 57 & 56.97 & 56.9648 & $\mathrm{CaOH}^{+}$ \\
\hline 73 & 73.055 & 73.0501 & $\left(\mathrm{H}_{2} \mathrm{O}\right)_{4} \mathrm{H}^{+}$ \\
\hline 73 & 73.055 & 73.0468 & $\mathrm{SiC}_{3} \mathrm{H}_{9}^{+}$ \\
\hline 103 & 103.06 & 103.0548 & $\mathrm{C}_{8} \mathrm{H}_{7}^{+}$ \\
\hline 133 & 133.07 & 133.0505 & $\mathrm{C}_{4} \mathrm{H}_{13} \mathrm{OSi}_{2}^{+}$ \\
\hline 147 & 147.06 & 147.0661 & $\left(\mathrm{CH}_{3}\right)_{3} \mathrm{SiOSi}\left(\mathrm{CH}_{3}\right)_{2}{ }^{+}$ \\
\hline 159 & 159.10 & 159.0293 & $\mathrm{C}_{6} \mathrm{H}_{7} \mathrm{O}_{5}^{+}$ \\
\hline 207 & 207.07 & 207.0324 & $\mathrm{C}_{5} \mathrm{H}_{15} \mathrm{O}_{3} \mathrm{Si}_{3}{ }^{+}$ \\
\hline 209 & 208.98 & 208.9798 & $\mathrm{Bi}^{+}$ \\
\hline 231 & 231.13 & 231.1749 & $\mathrm{C}_{16} \mathrm{H}_{23} \mathrm{O}^{+}$ \\
\hline 239 & 239.25 & 239.2375 & $\mathrm{C}_{16} \mathrm{H}_{31} \mathrm{O}^{+}$ \\
\hline 261 & 261.08 & 261.0238 & $\mathrm{C}_{15} \mathrm{H}_{11} \mathrm{Cl}_{2}^{+}$ \\
\hline 310 & 310.32 & 310.2872 & $\mathrm{C}_{20} \mathrm{H}_{38} \mathrm{O}_{2}^{+}$ \\
\hline 311 & 310.85 & 311.2950 & $\mathrm{C}_{20} \mathrm{H}_{39} \mathrm{O}_{2}^{+}$ \\
\hline 325 & 324.84 & 325.3107 & $\mathrm{C}_{21} \mathrm{H}_{41} \mathrm{O}_{2}^{+}$ \\
\hline 327 & 327.08 & 327.2324 & $\mathrm{C}_{22} \mathrm{H}_{31} \mathrm{O}_{2}^{+}$ \\
\hline 341 & 340.77 & 341.1025 & $\mathrm{C}_{19} \mathrm{H}_{17} \mathrm{O}_{6}^{+}$ \\
\hline 351 & 350.81 & 351.1808 & $\mathrm{C}_{19} \mathrm{H}_{27} \mathrm{O}_{6}^{+}$ \\
\hline 367 & 367.30 & 367.1757 & $\mathrm{C}_{19} \mathrm{H}_{27} \mathrm{O}_{7}^{+}$ \\
\hline 381 & 380.88 & 381.1913 & $\mathrm{C}_{20} \mathrm{H}_{29} \mathrm{O}_{7}^{+}$ \\
\hline 397 & 397.23 & 397.1862 & $\mathrm{C}_{20} \mathrm{H}_{29} \mathrm{O}_{8}^{+}$ \\
\hline 418 & $\mathrm{a}$ & 417.9602 & $\mathrm{Bi}_{2}^{+}$ \\
\hline 429 & 429.30 & 429.3733 & $\mathrm{C}_{29} \mathrm{H}_{49} \mathrm{O}_{2}^{+}$ \\
\hline 445 & 445.19 & 445.1737 & $\mathrm{C}_{23} \mathrm{H}_{27} \mathrm{NO}_{8}^{+}$ \\
\hline 453 & 453.13 & 453.1566 & $\mathrm{C}_{28} \mathrm{H}_{21} \mathrm{MgN}_{4} \mathrm{O}^{+}$ \\
\hline 461 & 461.10 & 461.1686 & $\mathrm{C}_{23} \mathrm{H}_{27} \mathrm{NO}_{9}^{+}$ \\
\hline 469 & 469.12 & 469.1515 & $\mathrm{C}_{28} \mathrm{H}_{21} \mathrm{MgN}_{4} \mathrm{O}_{2}^{+}$ \\
\hline 477 & 476.31 & 477.1635 & $\mathrm{C}_{23} \mathrm{H}_{27} \mathrm{NO}_{10}^{+}$ \\
\hline 485 & 485.04 & 485.1464 & $\mathrm{C}_{28} \mathrm{H}_{21} \mathrm{MgN}_{4} \mathrm{O}_{3}{ }^{+}$ \\
\hline 509 & 508.69 & 509.1464 & $\mathrm{C}_{30} \mathrm{H}_{21} \mathrm{MgN}_{4} \mathrm{O}_{3}{ }^{+}$ \\
\hline 541 & 541.33 & 541.4257 & $\mathrm{C}_{35} \mathrm{H}_{57} \mathrm{O}_{4}^{+}$ \\
\hline 565 & 565.35 & 565.4257 & $\mathrm{C}_{37} \mathrm{H}_{57} \mathrm{O}_{4}^{+}$ \\
\hline 597 & 597.35 & 597.4883 & $\mathrm{C}_{39} \mathrm{H}_{65} \mathrm{O}_{4}^{+}$ \\
\hline 627 & a & 626.9411 & $\mathrm{Bi}_{3}^{+}$ \\
\hline 643 & $\mathrm{a}$ & 642.936 & $\mathrm{Bi}_{3} \mathrm{O}^{+}$ \\
\hline 659 & a & 658.931 & $\mathrm{Bi}_{3} \mathrm{O}_{2}^{+}$ \\
\hline 691 & 691.55 & 690.6502 & $\mathrm{C}_{43} \mathrm{H}_{87} \mathrm{O}_{4} \mathrm{Na}^{+}$ \\
\hline 707 & 707.61 & 706.6451 & $\mathrm{C}_{43} \mathrm{H}_{87} \mathrm{O}_{5} \mathrm{Na}^{+}$ \\
\hline 723 & 722.497 & 722.64 & $\mathrm{C}_{43} \mathrm{H}_{87} \mathrm{O}_{6} \mathrm{Na}^{+}$ \\
\hline
\end{tabular}

a: No $\mathrm{m} / \mathrm{z}$ peaks were observed in the dry biofilm sample. 


\section{Supporting Information}

Table S2B. Dry biofilm sample observed mass to charge ratios in the negative ion mode.

\begin{tabular}{cccc}
\hline Unit mass $\mathrm{m} / \mathrm{z}$ & Observed $\mathrm{m} / \mathrm{z}$ & Theoretical $\mathrm{m} / \mathrm{z}$ & Possible assignment \\
\hline 26 & 26.01 & 26.0031 & $\mathrm{CN}^{-}$ \\
42 & 42.00 & 41.998 & $\mathrm{CNO}^{-}$ \\
63 & 62.97 & 62.9636 & $\mathrm{PO}_{2}^{-}$ \\
79 & 78.96 & 78.9585 & $\mathrm{PO}_{3}^{-}$ \\
137 & 136.92 & 136.9363 & $\mathrm{Si}_{2} \mathrm{O}_{5} \mathrm{H}^{-}$ \\
225 & 225.19 & 225.1855 & $\mathrm{C}_{2} \mathrm{H}_{3}\left(\mathrm{CH}_{2}\right)_{11} \mathrm{COO}^{-}$ \\
237 & 237.21 & 237.2218 & $\mathrm{C}_{16} \mathrm{H}_{29} \mathrm{O}^{-}$ \\
239 & 239.21 & 239.2375 & $\mathrm{C}_{16} \mathrm{H}_{31} \mathrm{O}^{-}$ \\
255 & 255.23 & 255.2324 & $\mathrm{C}_{16} \mathrm{H}_{31} \mathrm{O}_{2}^{-}$ \\
277 & 277.07 & 277.2168 & $\mathrm{C}_{18} \mathrm{H}_{29} \mathrm{O}_{2}^{-}$ \\
297 & 297.15 & 297.2794 & $\mathrm{C}_{19} \mathrm{H}_{37} \mathrm{O}_{2}^{-}$ \\
311 & 311.21 & 311.295 & $\mathrm{C}_{20} \mathrm{H}_{39} \mathrm{O}_{2}^{-}$ \\
325 & 324.07 & 325.3107 & $\mathrm{C}_{21} \mathrm{H}_{41} \mathrm{O}_{2}^{-}$ \\
339 & 338.83 & 339.3263 & $\mathrm{C}_{22} \mathrm{H}_{43} \mathrm{O}_{2}^{-}$ \\
341 & 341.05 & 341.1025 & $\mathrm{C}_{19} \mathrm{H}_{17} \mathrm{O}_{6}^{-}$ \\
381 & 380.79 & 381.1913 & $\mathrm{C}_{20} \mathrm{H}_{29} \mathrm{O}_{7}^{-}$ \\
421 & 421.14 & 421.1975 & $\mathrm{C}_{21} \mathrm{H}_{29} \mathrm{~N}_{2} \mathrm{O}_{7}^{-}$ \\
429 & 428.72 & 429.3733 & $\mathrm{C}_{29} \mathrm{H}_{49} \mathrm{O}_{2}^{-}$ \\
507 & 506.98 & 507.1671 & $\mathrm{C}_{31} \mathrm{H}_{23} \mathrm{MgN}_{4} \mathrm{O}_{2}^{-}$ \\
539 & 539.07 & 539.1934 & $\mathrm{C}_{32} \mathrm{H}_{27} \mathrm{MgN}_{4} \mathrm{O}_{3}^{-}$ \\
600 & $a$ & 599.5039 & $\mathrm{C}_{39} \mathrm{H}_{67} \mathrm{O}_{4}^{-}$ \\
610 & 610.21 & 610.4961 & $\mathrm{C}_{40} \mathrm{H}_{66} \mathrm{O}_{4}^{-}$ \\
611 & 611.11 & 611.2145 & $\mathrm{C}_{35} \mathrm{H}_{31} \mathrm{MgN}_{4} \mathrm{O}_{5}^{-}$ \\
\hline
\end{tabular}

${ }^{a}$ : No $\mathrm{m} / \mathrm{z}$ peaks were observed in the dry biofilm sample. 


\section{Supporting Information}

Table S3A. Dry MM1 medium sample observed mass to charge ratios in the positive ion mode.

\begin{tabular}{cccc}
\hline Unit mass $\mathrm{m} / \mathbf{z}$ & Observed $\mathrm{m} / \mathbf{z}$ & Theoretical m/z & Possible assignment \\
\hline 23 & 22.99 & 22.9892 & $\mathrm{Na}^{+}$ \\
28 & 27.98 & 27.9764 & $\mathrm{Si}^{+}$ \\
39 & 38.97 & 38.9632 & $\mathrm{~K}^{+}$ \\
40 & 39.96 & 39.962 & $\mathrm{Ca}^{+}$ \\
45 & 44.98 & 44.9793 & $\mathrm{SiOH}^{+}$ \\
57 & 56.97 & 56.9648 & $\mathrm{CaOH}^{+}$ \\
73 & 73.05 & 73.0468 & $\mathrm{SiC}_{3} \mathrm{H}_{9}{ }^{+}$ \\
133 & 133.07 & 133.0505 & $\mathrm{C}_{4} \mathrm{H}_{13} \mathrm{OSi}_{2}{ }^{+}$ \\
147 & 147.06 & 147.0661 & $\left.\left(\mathrm{CH}_{3}\right)_{3} \mathrm{SiOSi}_{3} \mathrm{CH}_{3}\right)_{2}^{+}$ \\
207 & 207.07 & 207.0324 & $\mathrm{C}_{5} \mathrm{H}_{15} \mathrm{O}_{3} \mathrm{Si}_{3}{ }^{+}$ \\
209 & 208.98 & 208.9798 & $\mathrm{Bi}^{+}$ \\
418 & $a$ & 417.9602 & $\mathrm{Bi}_{2}{ }^{+}$ \\
627 & $a$ & 626.9411 & $\mathrm{Bi}_{3}{ }^{+}$ \\
\hline
\end{tabular}

a: No $\mathrm{m} / \mathrm{z}$ peaks were observed in the dry biofilm sample.

Table S3B. Dry MM1 medium sample observed mass to charge ratios in the negative ion mode.

\begin{tabular}{cccc}
\hline Unit mass $\mathrm{m} / \mathrm{z}$ & Observed $\mathrm{m} / \mathrm{z}$ & Theoretical m/z & Possible assignment \\
\hline 26 & 26.01 & 26.0031 & $\mathrm{CN}^{-}$ \\
42 & 42.00 & 41.998 & $\mathrm{CNO}^{-}$ \\
63 & 62.97 & 62.9636 & $\mathrm{PO}_{2}^{-}$ \\
79 & 78.96 & 78.9585 & $\mathrm{PO}_{3}^{-}$ \\
137 & 136.92 & 136.9363 & $\mathrm{Si}_{2} \mathrm{O}_{5} \mathrm{H}^{-}$ \\
\hline
\end{tabular}




\section{Supporting Information}

Table S4A. Possible peak identification of representative peaks in the positive ion mode.

\begin{tabular}{|c|c|c|c|}
\hline $\mathrm{m} / \mathrm{z}$ & Possible assignment & Descriptions & Reference \\
\hline 23 & $\mathrm{Na}^{+}$ & Ion & 5 \\
\hline 28 & $\mathrm{Si}^{+} / \mathrm{C}_{2} \mathrm{H}_{4}^{+}$ & PDMS-related peaks & 6 \\
\hline 39 & $\mathrm{~K}^{+}$ & Ion & 5 \\
\hline 40 & $\mathrm{Ca}^{+}$ & Ion & 7 \\
\hline 45 & $\mathrm{C}_{2} \mathrm{H}_{5} \mathrm{O}^{+} / \mathrm{SiOH}^{+}$ & PDMS-related peaks & 8 \\
\hline \multirow[t]{2}{*}{57} & $\mathrm{Ca}(\mathrm{OH})^{+}$ & & 9 \\
\hline & $\mathrm{C}_{3} \mathrm{H}_{5} \mathrm{O}^{+}$ & & 10 \\
\hline 73 & $\mathrm{Si}_{2} \mathrm{OH}^{+} /\left(\mathrm{H}_{2} \mathrm{O}\right)_{4} \mathrm{H}^{+}$ & PDMS-related peaks/ Water cluster & 6 \\
\hline 103 & $\mathrm{C}_{8} \mathrm{H}_{7}^{+}$ & & 11 \\
\hline 133 & $\mathrm{C}_{4} \mathrm{H}_{13} \mathrm{OSi}_{2}^{+}$ & PDMS peaks & 12 \\
\hline 147 & $\left(\mathrm{CH}_{3}\right)_{3} \mathrm{SiOSi}\left(\mathrm{CH}_{3}\right)_{2}^{+}$ & PDMS peaks & 6 \\
\hline 159 & $\mathrm{C}_{6} \mathrm{H}_{7} \mathrm{O}_{5}^{+}$ & Polymer related peaks/fragment of $\beta$-D-Ethyl glucuronide & 13 \\
\hline $172^{\mathrm{b}}$ & $\mathrm{C}_{8} \mathrm{H}_{14} \mathrm{NO}_{3}^{+}$ & N-Acylhomoserine lactones (signal related) & 14,15 \\
\hline $175^{\mathrm{b}}$ & $\mathrm{C}_{10} \mathrm{H}_{9} \mathrm{NO}_{2}^{+}$ & Hormones/ Quinolone signal (QS) related & $16-18$ \\
\hline $188^{\mathrm{b}}$ & $\mathrm{C}_{11} \mathrm{H}_{10} \mathrm{NO}_{2}^{+} / \mathrm{C}_{8} \mathrm{H}_{14} \mathrm{NO}_{4}^{+}$ & Hormones/ Quinolone signal (QS) related & $16-18$ \\
\hline 207 & $\mathrm{C}_{7} \mathrm{H}_{21} \mathrm{O}_{2} \mathrm{Si}_{3}^{+}$ & PDMS peaks & 19,20 \\
\hline 209 & $\mathrm{Bi}^{+}$ & $\mathrm{Bi}_{3}{ }^{+}$primary ion beam related ions & 21 \\
\hline 221 & $\mathrm{C}_{6} \mathrm{H}_{17} \mathrm{O}_{3} \mathrm{Si}_{3}{ }^{+}$ & PDMS peaks & 19,20 \\
\hline 231 & $\mathrm{C}_{16} \mathrm{H}_{23} \mathrm{O}^{+}$ & Polymer & 22 \\
\hline $239^{\mathrm{b}}$ & $\mathrm{C}_{16} \mathrm{H}_{31} \mathrm{O}^{+}$ & Palmitic acid & 23,24 \\
\hline $243^{\mathrm{b}}$ & $\mathrm{C}_{12} \mathrm{H}_{11} \mathrm{~N}_{4} \mathrm{O}_{2}^{+}$ & Riboflavin (loss of ribityl side chain)/A-factor (Signal & $25-27$ \\
\hline $257^{\mathrm{b}}$ & $\mathrm{C}_{16} \mathrm{H}_{33} \mathrm{O}_{2}^{+}$ & Palmitic acid & 23,24 \\
\hline $260^{\mathrm{b}}$ & $\mathrm{C}_{16} \mathrm{H}_{22} \mathrm{NO}_{2}^{+}$ & Quinolone signal (QS) related & $16-18$ \\
\hline 295 & $\mathrm{C}_{8} \mathrm{H}_{23} \mathrm{O}_{4} \mathrm{Si}_{4}^{+}$ & PDMS peaks & 19,20 \\
\hline 297 & $\mathrm{C}_{19} \mathrm{H}_{37} \mathrm{O}_{2}^{+}$ & Monoacylglycerols/Anionic surfactants /Fatty acid & 28,29 \\
\hline 311 & $\mathrm{C}_{20} \mathrm{H}_{39} \mathrm{O}_{2}^{+}$ & Monoacylglycerols/Anionic surfactants /Fatty acid & 28,29 \\
\hline 325 & $\mathrm{C}_{21} \mathrm{H}_{41} \mathrm{O}_{2}^{+}$ & Monoacylglycerols/Anionic surfactants /Fatty acid & 28,29 \\
\hline 327 & $\mathrm{C}_{22} \mathrm{H}_{31} \mathrm{O}_{2}^{+}$ & Fatty acid; Docosohexaenoate (DHA); Aromatic surfactants & 29-31 \\
\hline 339 & $\mathrm{C}_{22} \mathrm{H}_{43} \mathrm{O}_{2}^{+}$ & Monoacylglycerols/Anionic surfactants /Fatty acid & 28,29 \\
\hline 341 & $\mathrm{C}_{19} \mathrm{H}_{17} \mathrm{O}_{6}^{+}$ & Fatty acid/Polymer & 29 \\
\hline $343^{\mathrm{b}}$ & $\mathrm{C}_{16} \mathrm{H}_{27} \mathrm{~N}_{2} \mathrm{O}_{6}{ }^{+}$ & N-Acylhomoserine lactones (signal related) & 14,15 \\
\hline 351 & $\mathrm{C}_{19} \mathrm{H}_{27} \mathrm{O}_{6}^{+}$ & Desmosterol/Dipalmitoyl phosphatidylcholine/Polymers/Fatty & 32 \\
\hline 353 & $\mathrm{C}_{23} \mathrm{H}_{45} \mathrm{O}_{2}^{+}$ & Monoacylglycerols/Anionic surfactants /fatty acid & 28,29 \\
\hline 367 & $\mathrm{C}_{19} \mathrm{H}_{27} \mathrm{O}_{7}^{+}$ & Polymers/ fatty acid & $33-36$ \\
\hline $377^{\mathrm{b}}$ & $\mathrm{C}_{17} \mathrm{H}_{21} \mathrm{~N}_{4} \mathrm{O}_{6}^{+}$ & Riboflavin & $25-27$ \\
\hline $378^{\mathrm{b}}$ & $\mathrm{C}_{17} \mathrm{H}_{22} \mathrm{~N}_{4} \mathrm{O}_{6}^{+}$ & Riboflavin & $25-27$ \\
\hline 381 & $\mathrm{C}_{20} \mathrm{H}_{29} \mathrm{O}_{7}^{+}$ & poly (styrene)/poly (4-vinyl phenol)/ fatty acid & 35,36 \\
\hline 397 & $\mathrm{C}_{20} \mathrm{H}_{29} \mathrm{O}_{8}^{+}$ & fatty acid side chain/ fatty acid & 35,36 \\
\hline 418 & $\mathrm{Bi}_{2}^{+}$ & $\mathrm{Bi}_{3}{ }^{+}$primary ion beam related ions & 21 \\
\hline 429 & $\mathrm{C}_{29} \mathrm{H}_{49} \mathrm{O}_{2}^{+}$ & Cyclic lipid (from $\alpha$-Tocopherol) & 37,38 \\
\hline 445 & $\mathrm{C}_{23} \mathrm{H}_{27} \mathrm{NO}_{8}^{+}$ & Glucuronides & 39 \\
\hline 453 & $\mathrm{C}_{28} \mathrm{H}_{21} \mathrm{MgN}_{4} \mathrm{O}^{+}$ & Cyclic lipid (from Chlorophyll a) & 37,38 \\
\hline 461 & $\mathrm{C}_{23} \mathrm{H}_{27} \mathrm{NO}_{9}^{+}$ & Glucuronides & 39 \\
\hline 469 & $\mathrm{C}_{28} \mathrm{H}_{21} \mathrm{MgN}_{4} \mathrm{O}_{2}^{+}$ & Cyclic lipid (from Chlorophyll a) & 37,38 \\
\hline 477 & $\mathrm{C}_{23} \mathrm{H}_{27} \mathrm{NO}_{10}^{+}$ & Glucuronides & 39 \\
\hline 485 & $\mathrm{C}_{28} \mathrm{H}_{21} \mathrm{MgN}_{4} \mathrm{O}_{3}^{+}$ & Cyclic lipid (from Chlorophyll a) & 37,38 \\
\hline 509 & $\mathrm{C}_{30} \mathrm{H}_{21} \mathrm{MgN}_{4} \mathrm{O}_{3}^{+}$ & waxy plant surfaces: triterpenoids and C34 fatty acid/ Cyclic & 37,38 \\
\hline $513^{\mathrm{b}}$ & $\mathrm{C}_{32} \mathrm{H}_{65} \mathrm{O}_{4}^{+}$ & Palmitic acid & 23 \\
\hline $519^{\mathrm{b}}$ & $\mathrm{C}_{32} \mathrm{H}_{43} \mathrm{~N}_{2} \mathrm{O}_{4}^{+}$ & Quinolone signal (QS) related & $16-18$ \\
\hline 541 & $\mathrm{C}_{35} \mathrm{H}_{57} \mathrm{O}_{4}^{+}$ & Microbial mat (diacylglycerol) & 37,38 \\
\hline 565 & $\mathrm{C}_{37} \mathrm{H}_{57} \mathrm{O}_{4}^{+}$ & Microbial mat (diacylglycerol) & 37,38 \\
\hline 597 & $\mathrm{C}_{39} \mathrm{H}_{65} \mathrm{O}_{4}^{+}$ & Microbial mat (diacylglycerol) & 37,38 \\
\hline 627 & $\mathrm{Bi}_{3}^{+}$ & $\mathrm{Bi}_{3}{ }^{+}$primary ion beam related ions & 21 \\
\hline 643 & $\mathrm{Bi}_{3} \mathrm{O}^{+}$ & $\mathrm{Bi}_{3}{ }^{+}$primary ion beam related ions & 21 \\
\hline 659 & $\mathrm{Bi}_{3} \mathrm{O}_{2}^{+}$ & $\mathrm{Bi}_{3}{ }^{+}$primary ion beam related ions & 21 \\
\hline 691 & $\mathrm{C}_{43} \mathrm{H}_{87} \mathrm{O}_{4} \mathrm{Na}^{+}$ & Glycerolipids; Hydroxyarchaeol & 40 \\
\hline
\end{tabular}


Supporting Information

$707 \quad \mathrm{C}_{43} \mathrm{H}_{87} \mathrm{O}_{5} \mathrm{Na}^{+} \quad$ Glycerolipids; Hydroxyarchaeol

$723 \quad \mathrm{C}_{43} \mathrm{H}_{87} \mathrm{O}_{6} \mathrm{Na}^{+} \quad$ Glycerolipids; Hydroxyarchaeol

Glycerolipids; Hydroxyarchaeol 40

b: The peaks were also identified by dry control samples, the related spectra were shown in Figure S4 


\section{Supporting Information}

Table S4B. Possible peak identification of representative ions in the negative ion mode.

\begin{tabular}{|c|c|c|c|}
\hline $\mathrm{m} / \mathrm{z}$ & Possible assignment & Descriptions & Reference \\
\hline 26 & $\mathrm{CN}^{-}$ & & 41,42 \\
\hline 42 & $\mathrm{CNO}^{-}$ & & 41 \\
\hline 63 & $\mathrm{PO}_{2}^{-}$ & & 43 \\
\hline 77 & $\left(\mathrm{SiO}_{2}\right) \mathrm{OH}^{-}$ & PDMS Interference peak & 44 \\
\hline 79 & $\mathrm{PO}_{3}^{-}$ & & 43 \\
\hline 137 & $\left(\mathrm{SiO}_{2}\right)_{2} \mathrm{OH}^{-}$ & PDMS Interference peak & 44 \\
\hline $170^{\mathrm{c}}$ & $\mathrm{C}_{8} \mathrm{H}_{12} \mathrm{NO}_{3}^{-}$ & $\mathrm{N}$-Acylhomoserine lactones (signal related) & 14,15 \\
\hline $173^{\mathrm{c}}$ & $\mathrm{C}_{10} \mathrm{H}_{7} \mathrm{NO}_{2}^{-}$ & Hormones/ Quinolone signal (QS) related & $16-18$ \\
\hline $186^{\mathrm{c}}$ & $\mathrm{C}_{8} \mathrm{H}_{12} \mathrm{NO}_{4}^{-}$ & Hormones/ Quinolone signal (QS) related/ N-Acylhomoserine lactones & $16-18$ \\
\hline 197 & $\left(\mathrm{SiO}_{2}\right)_{3} \mathrm{OH}^{-}$ & PDMS Interference peak & 44 \\
\hline 211 & {$\left[\mathrm{C}_{2} \mathrm{H}_{3}\left(\mathrm{CH}_{2}\right)_{10} \mathrm{COO}\right]-$} & Aliphatic chain fragment ions of fatty acids & 45 \\
\hline 225 & {$\left[\mathrm{C}_{2} \mathrm{H}_{3}\left(\mathrm{CH}_{2}\right)_{11} \mathrm{COO}\right]-$} & Aliphatic chain fragment ions of fatty acids & 45 \\
\hline $237^{\mathrm{c}}$ & $\mathrm{C}_{16} \mathrm{H}_{29} \mathrm{O}^{-}$ & Palmitic acid & 23,24 \\
\hline $241^{\mathrm{c}}$ & $\mathrm{C}_{12} \mathrm{H}_{9} \mathrm{~N}_{4} \mathrm{O}_{2}^{-}$ & Riboflavin/A-factor (Signal related)/ Diffusible signal factor & $25-27$ \\
\hline $255^{\mathrm{c}}$ & $\mathrm{C}_{16} \mathrm{H}_{31} \mathrm{O}_{2}^{-}$ & Palmitic acid/Fatty acid & $23,24,42$ \\
\hline 257 & $\left(\mathrm{SiO}_{2}\right)_{4} \mathrm{OH}^{-}$ & PDMS Interference peak & 44 \\
\hline $258^{\mathrm{c}}$ & $\mathrm{C}_{16} \mathrm{H}_{20} \mathrm{NO}_{2}^{-}$ & Hormones/ Quinolone signal (QS) related & $16-18$ \\
\hline 277 & $\mathrm{C}_{18} \mathrm{H}_{29} \mathrm{O}_{2}^{-}$ & C18 Fatty acid & 46 \\
\hline 297 & $\mathrm{C}_{19} \mathrm{H}_{37} \mathrm{O}_{2}^{-}$ & Fatty acid/ polar compounds/ $\mathrm{C}_{10}$ polar compounds & 47,48 \\
\hline 311 & $\mathrm{C}_{20} \mathrm{H}_{39} \mathrm{O}_{2}^{-}$ & Fatty acid/ polar compounds/ $\mathrm{C}_{11}$ polar compounds & 47,48 \\
\hline 325 & $\mathrm{C}_{21} \mathrm{H}_{41} \mathrm{O}_{2}^{-}$ & Fatty acid/ polar compounds/ $\mathrm{C}_{12}$ polar compounds & 47,48 \\
\hline 339 & $\mathrm{C}_{22} \mathrm{H}_{43} \mathrm{O}_{2}^{-}$ & Desorption of emulsifiers from polystyrene latexes; alkylbenzene & 47,48 \\
\hline $341^{\mathrm{c}}$ & $\mathrm{C}_{19} \mathrm{H}_{17} \mathrm{O}_{6}^{-}$ & ions of monoacylglyceryls of palmitic and stearic acid/ Hair; surface & 14,15 \\
\hline 381 & $\mathrm{C}_{20} \mathrm{H}_{29} \mathrm{O}_{7}^{-}$ & Fatty acid & 27,28 \\
\hline $376^{\mathrm{c}}$ & $\mathrm{C}_{17} \mathrm{H}_{20} \mathrm{~N}_{4} \mathrm{O}_{6}^{-}$ & Riboflavin & $25-27$ \\
\hline $377^{\mathrm{c}}$ & $\mathrm{C}_{17} \mathrm{H}_{21} \mathrm{~N}_{4} \mathrm{O}_{6}^{-}$ & Riboflavin & $25-27$ \\
\hline 421 & $\mathrm{C}_{30} \mathrm{H}_{61}{ }^{-} / \mathrm{C}_{20} \mathrm{H}_{27} \mathrm{~N}_{3} \mathrm{O}_{7}^{-}$ & Fragment of fibres/Protein drug release from biodegradable polymer & 49,50 \\
\hline 429 & $\mathrm{C}_{29} \mathrm{H}_{49} \mathrm{O}_{2-}$ & Alpha-Tocopherol $\left(\mathrm{C}_{29} \mathrm{H}_{50} \mathrm{O}_{2}\right) /$ Vitamin E/ Lipid & $37,38,51$ \\
\hline 507 & $\mathrm{C}_{31} \mathrm{H}_{23} \mathrm{MgN}_{4} \mathrm{O}_{2}^{-}$ & Cyclic lipid (from Chlorophyll a) & 37,38 \\
\hline 539 & $\mathrm{C}_{32} \mathrm{H}_{27} \mathrm{MgN}_{4} \mathrm{O}_{3}^{-}$ & Cyclic lipid (from Chlorophyll a) & 37,38 \\
\hline $511^{\mathrm{c}}$ & $\mathrm{C}_{32} \mathrm{H}_{63} \mathrm{O}_{4}^{-} / \mathrm{C}_{33} \mathrm{H}_{51} \mathrm{O}_{4}^{-}$ & Palmitic acid/Microbial mat (diacylglycerol) & 23 \\
\hline $517^{\mathrm{c}}$ & $\mathrm{C}_{32} \mathrm{H}_{41} \mathrm{~N}_{2} \mathrm{O}_{4}^{-}$ & Hormones/ Quinolone signal (QS) related & $16-18$ \\
\hline 600 & $\mathrm{C}_{39} \mathrm{H}_{67} \mathrm{O}_{4}^{-}$ & Microbial mat (diacylglycerol) & 37,38 \\
\hline 610 & $\mathrm{C}_{40} \mathrm{H}_{66} \mathrm{O}_{4}^{-}$ & Microbial mat (diacylglycerol) & 37,38 \\
\hline 611 & $\mathrm{C}_{35} \mathrm{H}_{31} \mathrm{MgN}_{4} \mathrm{O}_{5}^{-}$ & Cyclic lipid (from Chlorophyll a) & 37,38 \\
\hline
\end{tabular}

c: The peaks were also identified by dry control samples, the related spectra were shown in Figure S4 


\section{Supporting Information}

Table S5A. List of non-water cluster peaks selected in the positive spectral PCA.

\begin{tabular}{|c|c|c|c|}
\hline $\mathrm{m} / \mathrm{z}$ & Peak assignment & Descriptions and notes & Reference \\
\hline 23 & $\mathrm{Na}^{+}$ & $\mathrm{Na}^{+}$ & 5 \\
\hline 24 & $\mathrm{Mg}^{+}$ & $\mathrm{Mg}^{+}$ & 52 \\
\hline 27 & $\mathrm{C}_{2} \mathrm{H}_{3}^{+}$ & Polysaccharides & 53 \\
\hline 29 & $\mathrm{C}_{2} \mathrm{H}_{5}^{+}$ & Polysaccharides & 53 \\
\hline 30 & $\mathrm{CH}_{4} \mathrm{~N}^{+}$ & Glycine & 54,55 \\
\hline 39 & $\mathrm{~K}^{+}$ & $\mathrm{K}^{+}$ & 5 \\
\hline 40 & $\mathrm{Ca}^{+}$ & $\mathrm{Ca}^{+}$ & 56 \\
\hline 41 & $\mathrm{C}_{3} \mathrm{H}_{5}^{+}$ & Polysaccharides & 53 \\
\hline 44 & $\mathrm{C}_{2} \mathrm{H}_{6} \mathrm{~N}^{+}$ & Alanine & 54,55 \\
\hline 52 & $\mathrm{Cr}^{+}$ & $\mathrm{Cr}$ & 57 \\
\hline 55 & $\mathrm{C}_{4} \mathrm{H}_{7}^{+}$ & Polysaccharides & 53 \\
\hline 60 & $\mathrm{C}_{2} \mathrm{H}_{6} \mathrm{NO}^{+}$ & Serine & 54,55 \\
\hline 68 & $\mathrm{C}_{4} \mathrm{H}_{6} \mathrm{~N}^{+}$ & Proline & 54,55 \\
\hline 70 & $\mathrm{C}_{4} \mathrm{H}_{8} \mathrm{~N}^{+}$ & Proline & 54,55 \\
\hline 72 & $\mathrm{C}_{4} \mathrm{H}_{10} \mathrm{~N}^{+}$ & Valine & 54,55 \\
\hline 74 & $\mathrm{C}_{3} \mathrm{H}_{8} \mathrm{NO}^{+}$ & Threonine & 54,55 \\
\hline 82 & $\mathrm{C}_{4} \mathrm{H}_{6} \mathrm{~N}_{2}^{+}$ & Histidine & 54,55 \\
\hline 84 & $\mathrm{C}_{5} \mathrm{H}_{10} \mathrm{~N}^{+}$ & Lysine & 54,55 \\
\hline 86 & $\mathrm{C}_{4} \mathrm{H}_{8} \mathrm{NO}^{+} / \mathrm{C}_{5} \mathrm{H}_{12} \mathrm{~N}^{+}$ & Hydroxyproline/Leucine & 54,55 \\
\hline 100 & $\mathrm{C}_{4} \mathrm{H}_{10} \mathrm{~N}_{3}^{+}$ & Arginine & 54,55 \\
\hline 107 & $\mathrm{C}_{7} \mathrm{H}_{7} \mathrm{O}^{+}$ & Tyrosine & 54,55 \\
\hline 110 & $\mathrm{C}_{5} \mathrm{H}_{8} \mathrm{~N}_{3}^{+}$ & Histidine & 54,55 \\
\hline 118 & $\mathrm{C}_{8} \mathrm{H}_{8} \mathrm{~N}^{+}$ & Indole & 58 \\
\hline 120 & $\mathrm{C}_{8} \mathrm{H}_{10} \mathrm{~N}^{+}$ & Phenylalanine & 54,55 \\
\hline 130 & $\mathrm{C}_{9} \mathrm{H}_{8} \mathrm{~N}^{+}$ & Tryptophan & 54,55 \\
\hline 136 & $\mathrm{C}_{8} \mathrm{H}_{10} \mathrm{NO}^{+}$ & Tyrosine & 54,55 \\
\hline 159 & $\mathrm{C}_{7} \mathrm{H}_{11} \mathrm{O}_{4}^{+} / \mathrm{C}_{6} \mathrm{H}_{7} \mathrm{O}_{5}^{+}$ & Polymer/Protein/Fragment of $\beta$-D-Ethyl glucuronide & 59,60 \\
\hline $172^{\mathrm{d}}$ & $\mathrm{C}_{8} \mathrm{H}_{14} \mathrm{NO}_{3}^{+}$ & N-Acylhomoserine lactones (signal related) & 14,15 \\
\hline $175^{\mathrm{d}}$ & $\mathrm{C}_{10} \mathrm{H}_{9} \mathrm{NO}_{2}^{+}$ & Hormones/ Quinolone signal (QS) related/L-arginine & $16-18$ \\
\hline $188^{\mathrm{d}}$ & $\mathrm{C}_{11} \mathrm{H}_{10} \mathrm{NO}_{2}^{+} / \mathrm{C}_{8} \mathrm{H}_{14} \mathrm{NO}_{4}^{+}$ & $\begin{array}{l}\text { Hormones/ Quinolone signal (QS) related/ N- } \\
\text { Acylhomoserine lactones (signal related) }\end{array}$ & $16-18$ \\
\hline 199 & $\mathrm{C}_{12} \mathrm{H}_{23} \mathrm{O}_{2}^{+}$ & Diffusible signal factor family(DSF) & 50,61 \\
\hline 210 & $\mathrm{C}_{11} \mathrm{H}_{18} \mathrm{~N}_{2} \mathrm{O}_{2}^{+}$ & Diketopiperazines (DKPs), Cyclo (L-Leu-L-Val) & 50 \\
\hline 213 & $\mathrm{C}_{13} \mathrm{H}_{25} \mathrm{O}_{2}^{+}$ & Diffusible signal factor family(DSF) & 50,61 \\
\hline 214 & $\mathrm{C}_{10} \mathrm{H}_{16} \mathrm{NO}_{4}^{+}$ & N-Acylhomoserine lactones (signal related) & 14 \\
\hline $239^{d}$ & $\mathrm{C}_{16} \mathrm{H}_{31} \mathrm{O}^{+}$ & Palmitic acid & 23,24 \\
\hline $243^{\mathrm{d}}$ & $\begin{array}{l}\mathrm{C}_{12} \mathrm{H}_{11} \mathrm{~N}_{4} \mathrm{O}_{2}^{+} \\
/ \mathrm{C}_{13} \mathrm{H}_{23} \mathrm{O}_{4}^{+} / \mathrm{C}_{15} \mathrm{H}_{31} \mathrm{O}_{2}^{+}\end{array}$ & $\begin{array}{l}\text { Riboflavin (loss of ribityl side chain)/A-factor (Signal } \\
\text { related)/Diffusible signal factor family(DSF) }\end{array}$ & $25-27$ \\
\hline 245 & $\mathrm{C}_{14} \mathrm{H}_{17} \mathrm{~N}_{2} \mathrm{O}_{2}^{+}$ & Diketopiperazines (DKPs), Cyclo (L-Phe-L-Pro) & 50 \\
\hline $257^{\mathrm{d}}$ & $\mathrm{C}_{16} \mathrm{H}_{33} \mathrm{O}_{2}^{+}$ & Palmitic acid & 23,24 \\
\hline $260^{\mathrm{d}}$ & $\mathrm{C}_{16} \mathrm{H}_{22} \mathrm{NO}_{2}^{+}$ & Quinolone signal (QS) related & $16-18$ \\
\hline 261 & $\mathrm{C}_{15} \mathrm{H}_{11} \mathrm{Cl}_{2}^{+}$ & Chlorine-containing polymers & 10 \\
\hline 298 & $\mathrm{C}_{16} \mathrm{H}_{28} \mathrm{NO}_{4}^{+}$ & N-Acylhomoserine lactones (signal related) & 50 \\
\hline 327 & $\mathrm{C}_{21} \mathrm{H}_{27} \mathrm{O}_{3}^{+}$ & Fatty acid/ Microbial mat(monoacylglycerol) & 37,38 \\
\hline 341 & $\mathrm{C}_{19} \mathrm{H}_{17} \mathrm{O}_{6}^{+}$ & polymers & 62 \\
\hline $343^{\mathrm{d}}$ & $\mathrm{C}_{16} \mathrm{H}_{27} \mathrm{~N}_{2} \mathrm{O}_{6}^{+}$ & N-Acylhomoserine lactones (signal related) & 14,15 \\
\hline $377^{\mathrm{d}}$ & $\mathrm{C}_{17} \mathrm{H}_{21} \mathrm{~N}_{4} \mathrm{O}_{6}^{+}$ & Riboflavin & $25-27$ \\
\hline $378^{d}$ & $\mathrm{C}_{17} \mathrm{H}_{22} \mathrm{~N}_{4} \mathrm{O}_{6}^{+}$ & Riboflavin & $25-27$ \\
\hline 397 & $\mathrm{C}_{29} \mathrm{H}_{49}{ }^{+}$ & $\begin{array}{l}\text { Fatty acid side chain/ Cyclic lipid (from } 17 \alpha(\mathrm{H}), 21 \beta(\mathrm{H})- \\
\text { Norhopane) }\end{array}$ & 37,38 \\
\hline 413 & $\mathrm{C}_{28} \mathrm{H}_{45} \mathrm{O}_{2}^{+}$ & Cyclic lipid (from $\alpha$-Tocopherol) & 37,38 \\
\hline 429 & $\mathrm{C}_{29} \mathrm{H}_{49} \mathrm{O}_{2}^{+}$ & Cyclic lipid (from $\alpha$-Tocopherol) & 37,38 \\
\hline 445 & $\mathrm{C}_{23} \mathrm{H}_{27} \mathrm{NO}_{8}^{+}$ & Glucuronides & 39 \\
\hline
\end{tabular}




\section{Supporting Information}

\begin{tabular}{|c|c|c|c|}
\hline \multirow[t]{2}{*}{453} & \multicolumn{2}{|l|}{$\mathrm{C}_{18} \mathrm{H}_{30} \mathrm{~N}_{4} \mathrm{O}_{8} \mathrm{Na}^{+} /$} & \multirow[t]{2}{*}{37,38} \\
\hline & $\mathrm{C}_{28} \mathrm{H}_{21} \mathrm{MgN}_{4} \mathrm{O}^{+}$ & Polymer/Cyclic lipid (from Chlorophyll a) & \\
\hline 461 & $\mathrm{C}_{23} \mathrm{H}_{27} \mathrm{NO}_{9}^{+}$ & Glucuronides & 39 \\
\hline 469 & $\mathrm{C}_{28} \mathrm{H}_{21} \mathrm{MgN}_{4} \mathrm{O}_{2}^{+}$ & Cyclic lipid (from Chlorophyll a) & 37,38 \\
\hline 477 & $\mathrm{C}_{23} \mathrm{H}_{27} \mathrm{NO}_{10}{ }^{+}$ & Glucuronides & 39 \\
\hline 485 & $\mathrm{C}_{28} \mathrm{H}_{21} \mathrm{MgN}_{4} \mathrm{O}_{3}^{+}$ & Cyclic lipid (from Chlorophyll a) & 37,38 \\
\hline 509 & $\mathrm{C}_{32} \mathrm{H}_{61} \mathrm{O}_{4}^{+}$ & Microbial mat (diacylglycerol) & 37,38 \\
\hline $513^{\mathrm{d}}$ & $\mathrm{C}_{32} \mathrm{H}_{65} \mathrm{O}_{4}^{+}$ & Palmitic acid & 23 \\
\hline $519^{\mathrm{d}}$ & $\mathrm{C}_{32} \mathrm{H}_{43} \mathrm{~N}_{2} \mathrm{O}_{4}^{+}$ & Quinolone signal (QS) related & $16-18$ \\
\hline 525 & $\mathrm{C}_{34} \mathrm{H}_{53} \mathrm{O}_{4}^{+}$ & Microbial mat (diacylglycerol) & 37,38 \\
\hline 541 & $\mathrm{C}_{35} \mathrm{H}_{57} \mathrm{O}_{4}^{+}$ & Microbial mat (diacylglycerol) & 37,38 \\
\hline 565 & $\mathrm{C}_{36} \mathrm{H}_{69} \mathrm{O}_{4}^{+}$ & Microbial mat (diacylglycerol) & 37,38 \\
\hline 581 & $\mathrm{C}_{38} \mathrm{H}_{61} \mathrm{O}_{4}^{+}$ & Microbial mat (diacylglycerol) & 37,38 \\
\hline 597 & $\mathrm{C}_{39} \mathrm{H}_{65} \mathrm{O}_{4}^{+}$ & Microbial mat (diacylglycerol) & 37,38 \\
\hline 691 & $\mathrm{C}_{43} \mathrm{H}_{87} \mathrm{O}_{4}+\mathrm{Na}^{+}$ & Glycerolipids; Hydroxyarchaeol & 40 \\
\hline 707 & $\mathrm{C}_{43} \mathrm{H}_{87} \mathrm{O}_{5}+\mathrm{Na}^{+}$ & Glycerolipids; Hydroxyarchaeol & 40 \\
\hline 723 & $\mathrm{C}_{43} \mathrm{H}_{87} \mathrm{O}_{6}+\mathrm{Na}^{+}$ & Glycerolipids; Hydroxyarchaeol & 40 \\
\hline
\end{tabular}

d: The peaks were also identified by dry control samples, the related spectra were shown in Figure S4. 
Supporting Information

Table S5B. List of water cluster peaks selected in the positive spectral PCA.

\begin{tabular}{|c|c|c|c|}
\hline $\mathrm{m} / \mathrm{z}$ & Formula & Descriptions & Reference \\
\hline 19 & $\left(\mathrm{H}_{2} \mathrm{O}\right) \mathrm{H}^{+}$ & Water cluster & $63-66$ \\
\hline 37 & $\left(\mathrm{H}_{2} \mathrm{O}\right)_{2} \mathrm{H}^{+}$ & & \\
\hline 55 & $\left(\mathrm{H}_{2} \mathrm{O}\right)_{3} \mathrm{H}^{+}$ & & \\
\hline 73 & $\left(\mathrm{H}_{2} \mathrm{O}\right)_{4} \mathrm{H}^{+}$ & & \\
\hline 91 & $\left(\mathrm{H}_{2} \mathrm{O}\right)_{5} \mathrm{H}^{+}$ & & \\
\hline 109 & $\left(\mathrm{H}_{2} \mathrm{O}\right)_{6} \mathrm{H}^{+}$ & & \\
\hline 127 & $\left(\mathrm{H}_{2} \mathrm{O}\right)_{7} \mathrm{H}^{+}$ & & \\
\hline 145 & $\left(\mathrm{H}_{2} \mathrm{O}\right)_{8} \mathrm{H}^{+}$ & & \\
\hline 163 & $\left(\mathrm{H}_{2} \mathrm{O}\right){ }_{9} \mathrm{H}^{+}$ & & \\
\hline 181 & $\left(\mathrm{H}_{2} \mathrm{O}\right)_{10} \mathrm{H}^{+}$ & & \\
\hline 199 & $\left(\mathrm{H}_{2} \mathrm{O}\right)_{11} \mathrm{H}^{+}$ & & \\
\hline 217 & $\left(\mathrm{H}_{2} \mathrm{O}\right)_{12} \mathrm{H}^{+}$ & & \\
\hline 235 & $\left(\mathrm{H}_{2} \mathrm{O}\right)_{13} \mathrm{H}^{+}$ & & \\
\hline 253 & $\left(\mathrm{H}_{2} \mathrm{O}\right)_{14} \mathrm{H}^{+}$ & & \\
\hline 271 & $\left(\mathrm{H}_{2} \mathrm{O}\right)_{15} \mathrm{H}^{+}$ & & \\
\hline 289 & $\left(\mathrm{H}_{2} \mathrm{O}\right)_{16} \mathrm{H}^{+}$ & & \\
\hline 307 & $\left(\mathrm{H}_{2} \mathrm{O}\right)_{17} \mathrm{H}^{+}$ & & \\
\hline 325 & $\left(\mathrm{H}_{2} \mathrm{O}\right)_{18} \mathrm{H}^{+}$ & & \\
\hline 343 & $\left(\mathrm{H}_{2} \mathrm{O}\right)_{19} \mathrm{H}^{+}$ & & \\
\hline 361 & $\left(\mathrm{H}_{2} \mathrm{O}\right)_{20} \mathrm{H}^{+}$ & & \\
\hline 379 & $\left(\mathrm{H}_{2} \mathrm{O}\right)_{21} \mathrm{H}^{+}$ & & \\
\hline 397 & $\left(\mathrm{H}_{2} \mathrm{O}\right)_{22} \mathrm{H}^{+}$ & & \\
\hline 415 & $\left(\mathrm{H}_{2} \mathrm{O}\right)_{23} \mathrm{H}^{+}$ & & \\
\hline 433 & $\left(\mathrm{H}_{2} \mathrm{O}\right)_{24} \mathrm{H}^{+}$ & & \\
\hline 451 & $\left(\mathrm{H}_{2} \mathrm{O}\right)_{25} \mathrm{H}^{+}$ & & \\
\hline 469 & $\left(\mathrm{H}_{2} \mathrm{O}\right)_{26} \mathrm{H}^{+}$ & & \\
\hline 487 & $\left(\mathrm{H}_{2} \mathrm{O}\right)_{27} \mathrm{H}^{+}$ & & \\
\hline 505 & $\left(\mathrm{H}_{2} \mathrm{O}\right)_{28} \mathrm{H}^{+}$ & & \\
\hline 523 & $\left(\mathrm{H}_{2} \mathrm{O}\right)_{29} \mathrm{H}^{+}$ & & \\
\hline 541 & $\left(\mathrm{H}_{2} \mathrm{O}\right)_{30} \mathrm{H}^{+}$ & & \\
\hline 559 & $\left(\mathrm{H}_{2} \mathrm{O}\right)_{31} \mathrm{H}^{+}$ & & \\
\hline 577 & $\left(\mathrm{H}_{2} \mathrm{O}\right)_{32} \mathrm{H}^{+}$ & & \\
\hline 595 & $\left(\mathrm{H}_{2} \mathrm{O}\right)_{33} \mathrm{H}^{+}$ & & \\
\hline 613 & $\left(\mathrm{H}_{2} \mathrm{O}\right)_{34} \mathrm{H}^{+}$ & & \\
\hline 631 & $\left(\mathrm{H}_{2} \mathrm{O}\right)_{35} \mathrm{H}^{+}$ & & \\
\hline 649 & $\left(\mathrm{H}_{2} \mathrm{O}\right)_{36} \mathrm{H}^{+}$ & & \\
\hline 667 & $\left(\mathrm{H}_{2} \mathrm{O}\right)_{37} \mathrm{H}^{+}$ & & \\
\hline 685 & $\left(\mathrm{H}_{2} \mathrm{O}\right)_{38} \mathrm{H}^{+}$ & & \\
\hline 703 & $\left(\mathrm{H}_{2} \mathrm{O}\right)_{39} \mathrm{H}^{+}$ & & \\
\hline 721 & $\left(\mathrm{H}_{2} \mathrm{O}\right)_{40} \mathrm{H}^{+}$ & & \\
\hline 739 & $\left(\mathrm{H}_{2} \mathrm{O}\right)_{41} \mathrm{H}^{+}$ & & \\
\hline 757 & $\left(\mathrm{H}_{2} \mathrm{O}\right)_{42} \mathrm{H}^{+}$ & & \\
\hline 775 & $\left(\mathrm{H}_{2} \mathrm{O}\right)_{43} \mathrm{H}^{+}$ & & \\
\hline 793 & $\left(\mathrm{H}_{2} \mathrm{O}\right)_{44} \mathrm{H}^{+}$ & & \\
\hline
\end{tabular}




\section{Supporting Information}

Table S6A. List of non-water cluster peaks selected in the negative spectral PCA.

\begin{tabular}{|c|c|c|c|}
\hline $\mathrm{m} / \mathrm{z}$ & Tentative Formula & Description/Notes & Reference \\
\hline 71 & {$\left[\mathrm{C}_{2} \mathrm{H}_{3} \mathrm{COO}\right]^{-}$} & Aliphatic chain fragment ions of fatty acids & 35 \\
\hline 85 & {$\left[\mathrm{C}_{2} \mathrm{H}_{3}\left(\mathrm{CH}_{2}\right)_{1} \mathrm{COO}\right]^{-}$} & Aliphatic chain fragment ions of fatty acids & 35 \\
\hline 99 & {$\left[\mathrm{C}_{2} \mathrm{H}_{3}\left(\mathrm{CH}_{2}\right)_{2} \mathrm{COO}\right]^{-}$} & Aliphatic chain fragment ions of fatty acids & 35 \\
\hline 100 & $\mathrm{CrO}_{3}^{-}$ & Cr related peak & 67 \\
\hline 103 & $\mathrm{Cr}(\mathrm{OH})_{3}^{-}$ & Cr related peak & 67 \\
\hline 113 & {$\left[\mathrm{C}_{2} \mathrm{H}_{3}\left(\mathrm{CH}_{2}\right)_{3} \mathrm{COO}\right]^{-}$} & Aliphatic chain fragment ions of fatty acids & 35 \\
\hline 116 & $\mathrm{C}_{8} \mathrm{H}_{6} \mathrm{~N}^{-} / \mathrm{CrO}_{4}^{-}$ & Indole $/ \mathrm{Cr}$ related peak & 67 \\
\hline 120 & $\mathrm{Cr}(\mathrm{OH})_{3}(\mathrm{~s})+\mathrm{OH}^{-}$ & $\mathrm{Cr}$ related peak & 67 \\
\hline 127 & {$\left[\mathrm{C}_{2} \mathrm{H}_{3}\left(\mathrm{CH}_{2}\right)_{4} \mathrm{COO}\right]^{-}$} & Aliphatic chain fragment ions of fatty acids & 35 \\
\hline 138 & $\mathrm{Cr}(\mathrm{OH})_{3} \cdot \mathrm{H}_{2} \mathrm{O} \cdot \mathrm{OH}^{-}$ & Cr related peak & 67 \\
\hline 141 & {$\left[\mathrm{C}_{2} \mathrm{H}_{3}\left(\mathrm{CH}_{2}\right)_{5} \mathrm{COO}\right]^{-}$} & Aliphatic chain fragment ions of fatty acids & 35 \\
\hline 155 & {$\left[\mathrm{C}_{2} \mathrm{H}_{3}\left(\mathrm{CH}_{2}\right)_{6} \mathrm{COO}\right]^{-}$} & Aliphatic chain fragment ions of fatty acids & 35 \\
\hline 156 & $\mathrm{Cr}(\mathrm{OH})_{3} .2 \mathrm{H}_{2} \mathrm{O} . \mathrm{OH}^{-}$ & Cr related peak & 67 \\
\hline 169 & {$\left[\mathrm{C}_{2} \mathrm{H}_{3}\left(\mathrm{CH}_{2}\right)_{7} \mathrm{COO}\right]^{-}$} & Aliphatic chain fragment ions of fatty acids & 35 \\
\hline $170^{\mathrm{e}}$ & $\mathrm{C}_{8} \mathrm{H}_{12} \mathrm{NO}_{3}^{-}$ & N-Acylhomoserine lactones (signal related) & 14,15 \\
\hline $173^{\mathrm{e}}$ & $\mathrm{C}_{10} \mathrm{H}_{7} \mathrm{NO}_{2}^{-}$ & Hormones/ Quinolone signal (QS) related & $16-18$ \\
\hline 183 & {$\left[\mathrm{C}_{2} \mathrm{H}_{3}\left(\mathrm{CH}_{2}\right)_{8} \mathrm{COO}\right]^{-}$} & Aliphatic chain fragment ions of fatty acids & 35 \\
\hline $186^{\mathrm{e}}$ & $\begin{array}{l}\mathrm{C}_{8} \mathrm{H}_{12} \mathrm{NO}_{4}^{-} \\
/ \mathrm{C}_{11} \mathrm{H}_{8} \mathrm{NO}_{2}^{-}\end{array}$ & $\begin{array}{l}\text { Hormones/ Quinolone signal (QS) related/ N-Acylhomoserine } \\
\text { lactones (signal related) }\end{array}$ & $16-18$ \\
\hline 199 & $\mathrm{C}_{12} \mathrm{H}_{23} \mathrm{O}_{2}^{-}$ & Lauric acid & 45 \\
\hline 209 & $\mathrm{C}_{11} \mathrm{H}_{17} \mathrm{~N}_{2} \mathrm{O}_{2}^{-}$ & Diketopiperazines (DKPs), Cyclo (L-Leu-L-Val) & 50,61 \\
\hline 211 & $\begin{array}{l}{\left[\mathrm{C}_{2} \mathrm{H}_{3}\left(\mathrm{CH}_{2}\right){ }_{10} \mathrm{COO}^{-}\right.} \\
/ \mathrm{C}_{13} \mathrm{H}_{23} \mathrm{O}_{2}^{-}\end{array}$ & $\begin{array}{l}\text { Aliphatic chain fragment ions of fatty acids/ Diffusible signal } \\
\text { factor family(DSF) }\end{array}$ & 50,61 \\
\hline 212 & $\mathrm{C}_{10} \mathrm{H}_{14} \mathrm{NO}_{4}^{-}$ & N-Acylhomoserine lactones (signal related) & 14 \\
\hline 213 & $\mathrm{CH}_{3}\left(\mathrm{CH}_{2}\right)_{11} \mathrm{COO}^{-}$ & Tridecylic acid & 45 \\
\hline 216 & $\mathrm{Cr}_{2} \mathrm{O}_{7}^{-}$ & Cr related peak & 67 \\
\hline 225 & {$\left[\mathrm{C}_{2} \mathrm{H}_{3}\left(\mathrm{CH}_{2}\right)_{11} \mathrm{COO}^{-}\right.$} & Aliphatic chain fragment ions of fatty acids & 35 \\
\hline 227 & $\mathrm{C}_{14} \mathrm{H}_{27} \mathrm{O}_{2}^{-}$ & Myristic acid & 45 \\
\hline $237^{\mathrm{e}}$ & $\mathrm{C}_{16} \mathrm{H}_{29} \mathrm{O}^{-}$ & Palmitic acid & 23,24 \\
\hline 239 & {$\left[\mathrm{C}_{2} \mathrm{H}_{3}\left(\mathrm{CH}_{2}\right)_{12} \mathrm{COO}\right]^{-}$} & Aliphatic chain fragment ions of fatty acids & 35 \\
\hline $241^{\mathrm{e}}$ & $\begin{array}{l}\mathrm{C}_{12} \mathrm{H}_{9} \mathrm{~N}_{4} \mathrm{O}_{2}^{-} \\
/ \mathrm{C}_{13} \mathrm{H}_{21} \mathrm{O}_{4}^{-} \\
/ \mathrm{C}_{15} \mathrm{H}_{29} \mathrm{O}_{2}^{-}\end{array}$ & $\begin{array}{l}\text { Pentadecylic acid/Riboflavin/A-factor (Signal related)/ } \\
\text { Diffusible signal factor family(DSF) }\end{array}$ & $25-27,45,50,61$ \\
\hline 243 & $\mathrm{C}_{14} \mathrm{H}_{15} \mathrm{~N}_{2} \mathrm{O}_{2}^{-}$ & Diketopiperazines (DKPs), Cyclo (L-Phe-L-Pro) & 50,61 \\
\hline 249 & $\mathrm{C}_{16} \mathrm{H}_{25} \mathrm{O}_{2}^{-}$ & Fatty acid & 37,38 \\
\hline $255^{\mathrm{e}}$ & $\mathrm{C}_{16} \mathrm{H}_{31} \mathrm{O}_{2}^{-}$ & Palmitic acid/Fatty acid & $23,24,45$ \\
\hline $258^{\mathrm{e}}$ & $\mathrm{C}_{16} \mathrm{H}_{20} \mathrm{NO}_{2}^{-}$ & Hormones/ Quinolone signal (QS) related & $16-18$ \\
\hline 265 & $\mathrm{C}_{17} \mathrm{H}_{29} \mathrm{O}_{2}^{-}$ & Fatty acid & 37,38 \\
\hline 277 & $\mathrm{C}_{18} \mathrm{H}_{29} \mathrm{O}_{2}^{-}$ & Fatty acid & $37,38,51$ \\
\hline 296 & $\mathrm{C}_{16} \mathrm{H}_{26} \mathrm{NO}_{4}^{-}$ & N-Acylhomoserine lactones (signal related) & 14 \\
\hline 297 & $\mathrm{C}_{19} \mathrm{H}_{37} \mathrm{O}_{2}^{-}$ & Fatty acid/ polar compounds & 47,48 \\
\hline 311 & $\mathrm{C}_{20} \mathrm{H}_{39} \mathrm{O}_{2}^{-}$ & Fatty acid/ polar compounds & 47,48 \\
\hline 317 & $\mathrm{C}_{21} \mathrm{H}_{33} \mathrm{O}_{2}^{-}$ & Polymer, lipids or protein related peaks & $37,38,68$ \\
\hline 325 & $\mathrm{C}_{21} \mathrm{H}_{41} \mathrm{O}_{2}^{-}$ & Fatty acid/ polar compounds & 47,48 \\
\hline 333 & $\mathrm{C}_{21} \mathrm{H}_{33} \mathrm{O}_{3}^{-}$ & Microbial mat (monoacylglycerol) & 37,38 \\
\hline 339 & $\mathrm{C}_{22} \mathrm{H}_{43} \mathrm{O}_{2}^{-}$ & Fatty acid/ polar compounds & 47,48 \\
\hline $341^{\mathrm{e}}$ & $\begin{array}{l}\mathrm{C}_{16} \mathrm{H}_{25} \mathrm{~N}_{2} \mathrm{O}_{6}^{-} \\
/ \mathrm{C}_{21} \mathrm{H}_{41} \mathrm{O}_{3}^{-}\end{array}$ & N-Acylhomoserine lactones (signal related)/Fatty acid/polymer & 14,15 \\
\hline $376^{\mathrm{e}}$ & $\mathrm{C}_{17} \mathrm{H}_{20} \mathrm{~N}_{4} \mathrm{O}_{6}^{-}$ & Riboflavin & $25-27$ \\
\hline $377^{\mathrm{e}}$ & $\mathrm{C}_{17} \mathrm{H}_{21} \mathrm{~N}_{4} \mathrm{O}_{6}^{-}$ & Riboflavin & $25-27$ \\
\hline 419 & $\mathrm{C}_{29} \mathrm{H}_{39} \mathrm{O}_{2}^{-}$ & Cyclic lipid (from $\alpha$-Tocopherol) & 37,38 \\
\hline 429 & $\mathrm{C}_{29} \mathrm{H}_{49} \mathrm{O}_{2}^{-}$ & Cyclic lipid (from $\alpha$-Tocopherol) & 37,38 \\
\hline 433 & $\mathrm{C}_{32} \mathrm{H}_{49^{-}}$ & Cyclic lipid (from $\beta$ - $\beta$-Carotene) & 37,38 \\
\hline 445 & $\mathrm{C}_{33} \mathrm{H}_{49}^{-}$ & Cyclic lipid (from $\beta$ - $\beta$-Carotene) & 37,38 \\
\hline 447 & $\mathrm{C}_{33} \mathrm{H}_{51}^{-}$ & Cyclic lipid (from $\beta$ - $\beta$-Carotene) & 37,38 \\
\hline 453 & $\mathrm{C}_{28} \mathrm{H}_{21} \mathrm{MgN}_{4} \mathrm{O}^{-}$ & Cyclic lipid (from Chlorophyll a) & 37,38 \\
\hline
\end{tabular}




\section{Supporting Information}

\begin{tabular}{|c|c|c|c|}
\hline 455 & $\mathrm{C}_{17} \mathrm{H}_{20} \mathrm{~N}_{4} \mathrm{O}_{9} \mathrm{P}^{-}$ & Flavin mononucleotide (FMN) & 69 \\
\hline 481 & $\mathrm{C}_{30} \mathrm{H}_{25} \mathrm{MgN}_{4} \mathrm{O}^{-}$ & Cyclic lipid (from Chlorophyll a) & 37,38 \\
\hline 497 & $\mathrm{C}_{30} \mathrm{H}_{25} \mathrm{MgN}_{4} \mathrm{O}_{2}^{-}$ & Cyclic lipid (from Chlorophyll a) & 37,38 \\
\hline $511^{\mathrm{e}}$ & $\mathrm{C}_{32} \mathrm{H}_{63} \mathrm{O}_{4}^{-} / \mathrm{C}_{33} \mathrm{H}_{51} \mathrm{O}_{4}^{-}$ & Palmitic acid/Microbial mat (diacylglycerol) & 23 \\
\hline $517^{\mathrm{e}}$ & $\mathrm{C}_{32} \mathrm{H}_{41} \mathrm{~N}_{2} \mathrm{O}_{4}^{-}$ & Hormones/ Quinolone signal (QS) related & $16-18$ \\
\hline 525 & $\mathrm{C}_{34} \mathrm{H}_{53} \mathrm{O}_{4}^{-}$ & Microbial mat (diacylglycerol) & 37,38 \\
\hline 555 & $\mathrm{C}_{36} \mathrm{H}_{59} \mathrm{O}_{4}^{-}$ & Microbial mat (diacylglycerol) & 37,38 \\
\hline 571 & $\mathrm{C}_{37} \mathrm{H}_{63} \mathrm{O}_{4}^{-}$ & Microbial mat (diacylglycerol) & 37,38 \\
\hline 600 & $\mathrm{C}_{39} \mathrm{H}_{68} \mathrm{O}_{4}^{-}$ & Microbial mat (diacylglycerol) & 37,38 \\
\hline 610 & $\mathrm{C}_{40} \mathrm{H}_{66} \mathrm{O}_{4}^{-}$ & Microbial mat (diacylglycerol) & 37,38 \\
\hline 611 & $\mathrm{C}_{35} \mathrm{H}_{31} \mathrm{MgN}_{4} \mathrm{O}_{5}^{-}$ & Cyclic lipid (from Chlorophyll a) & 37,38 \\
\hline 624 & $\mathrm{C}_{41} \mathrm{H}_{68} \mathrm{O}_{4}^{-}$ & Microbial mat (diacylglycerol) & 37,38 \\
\hline 627 & $\mathrm{C}_{35} \mathrm{H}_{31} \mathrm{MgN}_{4} \mathrm{O}_{6}^{-}$ & Cyclic lipid (from Chlorophyll a) & 37,38 \\
\hline 638 & $\mathrm{C}_{41} \mathrm{H}_{50} \mathrm{O}_{6}^{-}$ & Microbial mat (carotenoids) & 37,38 \\
\hline 643 & $\mathrm{C}_{41} \mathrm{H}_{55} \mathrm{O}_{6}^{-}$ & Microbial mat (carotenoids) & 37,38 \\
\hline 651 & $\mathrm{C}_{42} \mathrm{H}_{51} \mathrm{O}_{6}^{-}$ & Microbial mat (carotenoids) & 37,38 \\
\hline 652 & $\mathrm{C}_{42} \mathrm{H}_{52} \mathrm{O}_{6}^{-}$ & Microbial mat (carotenoids) & 37,38 \\
\hline 667 & $\mathrm{C}_{43} \mathrm{H}_{55} \mathrm{O}_{6}^{-}$ & Microbial mat (carotenoids) & 37,38 \\
\hline 680 & $\mathrm{C}_{44} \mathrm{H}_{56} \mathrm{O}_{6}^{-}$ & Microbial mat (carotenoids) & 37,38 \\
\hline 683 & $\mathrm{C}_{44} \mathrm{H}_{59} \mathrm{O}_{6}^{-}$ & Microbial mat (carotenoids) & 37,38 \\
\hline 694 & $\mathrm{C}_{45} \mathrm{H}_{58} \mathrm{O}_{6}^{-}$ & Microbial mat (carotenoids) & 37,38 \\
\hline 708 & $\mathrm{C}_{46} \mathrm{H}_{60} \mathrm{O}_{6}^{-}$ & Microbial mat (carotenoids) & 37,38 \\
\hline 723 & $\mathrm{C}_{47} \mathrm{H}_{63} \mathrm{O}_{6}^{-}$ & Microbial mat (carotenoids) & 37,38 \\
\hline 739 & $\mathrm{C}_{48} \mathrm{H}_{67} \mathrm{O}_{6}^{-}$ & Microbial mat (triacylglycerol) & 37,38 \\
\hline 744 & $\mathrm{C}_{48} \mathrm{H}_{71} \mathrm{O}_{6}^{-}$ & Microbial mat (triacylglycerol) & 37,38 \\
\hline 755 & $\mathrm{C}_{49} \mathrm{H}_{70} \mathrm{O}_{6}^{-}$ & Microbial mat (triacylglycerol) & 37,38 \\
\hline
\end{tabular}

e: The peaks were also identified by dry control samples, the related spectra were shown in Figure S4. 


\section{Supporting Information}

Table S6B. List of water cluster peaks selected in the negative spectral PCA.

\begin{tabular}{|c|c|c|c|}
\hline Mass/Charge & Formula & Descriptions & Reference \\
\hline 17 & $\mathrm{OH}^{-}$ & Water cluster & $63-66$ \\
\hline 35 & $\mathrm{H}_{2} \mathrm{OOH}^{-}$ & & \\
\hline 53 & $\left(\mathrm{H}_{2} \mathrm{O}\right)_{2} \mathrm{OH}^{-}$ & & \\
\hline 71 & $\left(\mathrm{H}_{2} \mathrm{O}\right)_{3} \mathrm{OH}^{-}$ & & \\
\hline 89 & $\left(\mathrm{H}_{2} \mathrm{O}\right)_{4} \mathrm{OH}^{-}$ & & \\
\hline 107 & $\left(\mathrm{H}_{2} \mathrm{O}\right)_{5} \mathrm{OH}^{-}$ & & \\
\hline 125 & $\left(\mathrm{H}_{2} \mathrm{O}\right)_{6} \mathrm{OH}^{-}$ & & \\
\hline 143 & $\left(\mathrm{H}_{2} \mathrm{O}\right)_{7} \mathrm{OH}^{-}$ & & \\
\hline 161 & $\left(\mathrm{H}_{2} \mathrm{O}\right)_{8} \mathrm{OH}^{-}$ & & \\
\hline 179 & $\left(\mathrm{H}_{2} \mathrm{O}\right)_{9} \mathrm{OH}^{-}$ & & \\
\hline 197 & $\left(\mathrm{H}_{2} \mathrm{O}\right)_{10} \mathrm{OH}^{-}$ & & \\
\hline 215 & $\left(\mathrm{H}_{2} \mathrm{O}\right)_{11} \mathrm{OH}^{-}$ & & \\
\hline 233 & $\left(\mathrm{H}_{2} \mathrm{O}\right)_{12} \mathrm{OH}^{-}$ & & \\
\hline 251 & $\left(\mathrm{H}_{2} \mathrm{O}\right)_{13} \mathrm{OH}^{-}$ & & \\
\hline 269 & $\left(\mathrm{H}_{2} \mathrm{O}\right)_{14} \mathrm{OH}^{-}$ & & \\
\hline 287 & $\left(\mathrm{H}_{2} \mathrm{O}\right)_{15} \mathrm{OH}^{-}$ & & \\
\hline 305 & $\left(\mathrm{H}_{2} \mathrm{O}\right)_{16} \mathrm{OH}^{-}$ & & \\
\hline 323 & $\left(\mathrm{H}_{2} \mathrm{O}\right)_{17} \mathrm{OH}^{-}$ & & \\
\hline 341 & $\left(\mathrm{H}_{2} \mathrm{O}\right)_{18} \mathrm{OH}^{-}$ & & \\
\hline 359 & $\left(\mathrm{H}_{2} \mathrm{O}\right)_{19} \mathrm{OH}^{-}$ & & \\
\hline 377 & $\left(\mathrm{H}_{2} \mathrm{O}\right)_{20} \mathrm{OH}^{-}$ & & \\
\hline 395 & $\left(\mathrm{H}_{2} \mathrm{O}\right)_{21} \mathrm{OH}^{-}$ & & \\
\hline 413 & $\left(\mathrm{H}_{2} \mathrm{O}\right)_{22} \mathrm{OH}^{-}$ & & \\
\hline 431 & $\left(\mathrm{H}_{2} \mathrm{O}\right)_{23} \mathrm{OH}^{-}$ & & \\
\hline 449 & $\left(\mathrm{H}_{2} \mathrm{O}\right)_{24} \mathrm{OH}^{-}$ & & \\
\hline 467 & $\left(\mathrm{H}_{2} \mathrm{O}\right)_{25} \mathrm{OH}^{-}$ & & \\
\hline 485 & $\left(\mathrm{H}_{2} \mathrm{O}\right)_{26} \mathrm{OH}^{-}$ & & \\
\hline 503 & $\left(\mathrm{H}_{2} \mathrm{O}\right)_{27} \mathrm{OH}^{-}$ & & \\
\hline 521 & $\left(\mathrm{H}_{2} \mathrm{O}\right)_{28} \mathrm{OH}^{-}$ & & \\
\hline 539 & $\left(\mathrm{H}_{2} \mathrm{O}\right)_{29} \mathrm{OH}^{-}$ & & \\
\hline 557 & $\left(\mathrm{H}_{2} \mathrm{O}\right)_{30} \mathrm{OH}^{-}$ & & \\
\hline 575 & $\left(\mathrm{H}_{2} \mathrm{O}\right)_{31} \mathrm{OH}^{-}$ & & \\
\hline 593 & $\left(\mathrm{H}_{2} \mathrm{O}\right)_{32} \mathrm{OH}^{-}$ & & \\
\hline 611 & $\left(\mathrm{H}_{2} \mathrm{O}\right)_{33} \mathrm{OH}^{-}$ & & \\
\hline 629 & $\left(\mathrm{H}_{2} \mathrm{O}\right)_{34} \mathrm{OH}^{-}$ & & \\
\hline 647 & $\left(\mathrm{H}_{2} \mathrm{O}\right)_{35} \mathrm{OH}^{-}$ & & \\
\hline 665 & $\left(\mathrm{H}_{2} \mathrm{O}\right)_{36} \mathrm{OH}^{-}$ & & \\
\hline 683 & $\left(\mathrm{H}_{2} \mathrm{O}\right)_{37} \mathrm{OH}^{-}$ & & \\
\hline 701 & $\left(\mathrm{H}_{2} \mathrm{O}\right)_{38} \mathrm{OH}^{-}$ & & \\
\hline 719 & $\left(\mathrm{H}_{2} \mathrm{O}\right)_{39} \mathrm{OH}^{-}$ & & \\
\hline 737 & $\left(\mathrm{H}_{2} \mathrm{O}\right)_{40} \mathrm{OH}^{-}$ & & \\
\hline 755 & $\left(\mathrm{H}_{2} \mathrm{O}\right)_{41} \mathrm{OH}^{-}$ & & \\
\hline 773 & $\left(\mathrm{H}_{2} \mathrm{O}\right)_{42} \mathrm{OH}^{-}$ & & \\
\hline 791 & $\left(\mathrm{H}_{2} \mathrm{O}\right)_{43} \mathrm{OH}^{-}$ & & \\
\hline
\end{tabular}




\section{Supporting Information}

\section{List of movies}

Movies of representative 3D images are provided to facilitate visualization of the key biofilm EPS components. These components correspond to the 3D images in Figure 4C and Figure 4F. Two movies are provided as the following:

Movie 1 (si_002) showing characteristic $\mathrm{m} / \mathrm{z}$ peaks of the untreated biofilm sample in the negative ion mode (Figure 4F) consisting of the EPS matrix m/z $241,429,445$; QS signal and fatty acid $\mathrm{m} / \mathrm{z}^{-} 173$, 186, 255; and Cr reduction related $\mathrm{m} / \mathrm{z}^{-} 100,120,216$, respectively. Similarly, corresponding $\mathrm{m} / \mathrm{z}$ peaks in the Biofilm $+\mathrm{Cr}$ sample in the negative mode (Figure 4F) in the following: the EPS matrix m/ $\mathrm{z}^{-} 241$, 429, 445; QS signal and fatty acid $\mathrm{m} / \mathrm{z}^{-} 173,186,255$; and $\mathrm{Cr}$ reduction related $\mathrm{m} / \mathrm{z}^{-} 100,120,216$, respectively. The color legend is listed in Table S7A for convenience of viewing.

Table S7A. List of the positive $\mathrm{m} / \mathrm{z}$ peaks in the 3D movie si_002

\begin{tabular}{llll}
\hline $\mathrm{m} / \mathrm{z}$ & Possible formula & Descriptions & Color legend \\
\hline 100 & $\mathrm{CrO}_{3}^{-}$ & Cr related & $\mathrm{I}$ \\
120 & $\mathrm{Cr}(\mathrm{OH})_{3} \mathrm{OH}^{-}$ & Cr related & $\mathrm{I}$ \\
173 & $\mathrm{C}_{10} \mathrm{H}_{7} \mathrm{NO}_{2}^{-}$ & $\mathrm{QS}$ & $\mathrm{I}$ \\
186 & $\mathrm{C}_{11} \mathrm{H}_{8} \mathrm{NO}_{2}^{-}$ & $\mathrm{QS}$ & $\mathrm{I}$ \\
216 & $\mathrm{Cr}_{2} \mathrm{O}_{7}^{-}$ & Cr related & $\mathrm{I}$ \\
241 & $\mathrm{C}_{12} \mathrm{H}_{9} \mathrm{~N}_{4} \mathrm{O}_{2}^{-}$ & Riboflavin & $\mathrm{I}$ \\
255 & $\mathrm{C}_{16} \mathrm{H}_{31} \mathrm{O}_{2}^{-}$ & Palmitic Acid & $\mathrm{I}$ \\
429 & $\mathrm{C}_{29} \mathrm{H}_{49} \mathrm{O}_{2}^{-}$ & Lipid & $\mathrm{I}$ \\
445 & $\mathrm{C}_{30} \mathrm{H}_{53} \mathrm{O}_{2}^{-}$ & Lipid & $\mathrm{I}$ \\
\hline
\end{tabular}




\section{Supporting Information}

Movie 2 (si_003) showing characteristic $\mathrm{m} / \mathrm{z}$ peaks of the untreated biofilm sample in the positive ion mode (Figure 4C) consisting of water clusters $\mathrm{m} / \mathrm{z}^{+} 109,253,325$; polymer fragments $\mathrm{m} / \mathrm{z}^{+} 159,261,341$; and glucuronide fragments $\mathrm{m} / \mathrm{z}^{+} 445,461,477$, respectively. Similarly corresponding $\mathrm{m} / \mathrm{z}$ peaks in the Biofilm $+\mathrm{Cr}$ sample in the positive mode (Figure 4C) in the following: water clusters $\mathrm{m} / \mathrm{z}^{+} 109,253,325$; polymer fragments $\mathrm{m} / \mathrm{z}^{+} 159,261,341$; and glucuronide fragments $\mathrm{m} / \mathrm{z}^{+} 445,461,477$, respectively are also illustrated in Movie 2 (si_003). The color legend is listed in Table S7B for convenience of viewing.

Table S7B. List of the positive $\mathrm{m} / \mathrm{z}$ peaks in the 3D movie in si_003

\begin{tabular}{llll}
\hline $\mathrm{m} / \mathrm{z}$ & Possible formula & Descriptions & Color legend \\
\hline 109 & $(\mathrm{H} 2 \mathrm{O})_{6} \mathrm{H}^{+}$ & Water cluster & $\mathrm{I}$ \\
159 & $\mathrm{C}_{6} \mathrm{H}_{7} \mathrm{O}_{5}{ }^{+}$ & Polymer & $\mathrm{I}$ \\
253 & $\left(\mathrm{H}_{2} \mathrm{O}\right)_{14} \mathrm{H}^{+}$ & Water cluster & $\mathrm{I}$ \\
261 & $\mathrm{C}_{15} \mathrm{H}_{11} \mathrm{Cl}_{2}{ }^{+}$ & Polymer & $\mathrm{I}$ \\
325 & $\left(\mathrm{H}_{2} \mathrm{O}\right) 1_{8} \mathrm{H}^{+}$ & Water cluster & $\mathrm{I}$ \\
341 & $\mathrm{C}_{19} \mathrm{H}_{17} \mathrm{O}_{6}{ }^{+}$ & Polymer & $\mathrm{I}$ \\
445 & $\mathrm{C}_{23} \mathrm{H}_{27} \mathrm{NO}_{8}{ }^{+}$ & Glucuronide & $\mathrm{I}$ \\
461 & $\mathrm{C}_{23} \mathrm{H}_{27} \mathrm{NO}_{9}{ }^{+}$ & Glucuronide & $\mathrm{I}$ \\
477 & $\mathrm{C}_{23} \mathrm{H}_{27} \mathrm{NO}_{10}{ }^{+}$ & Glucuronide & $\mathrm{I}$ \\
\hline
\end{tabular}




\section{Supporting Information}

\section{References}

(1) Yang, L.; Zhu, Z. H.; Yu, X. Y.; Rodek, E.; Saraf, L.; Thevuthasan, T.; Cowin, J. P. Surf Interface Anal 2014, 46, 224-228.

(2) Yu, J. C.; Zhou, Y. F.; Hua, X.; Zhu, Z. H.; Yu, X. Y. Jove-J Vis Exp 2016.

(3) Zhou, Y.; Yao, J.; Ding, Y.; Yu, J.; Hua, X.; Evans, J. E.; Yu, X.; Lao, D. B.; Heldebrant, D. J.; Nune, S. K.; Cao, B.; Bowden, M. E.; Yu, X. Y.; Wang, X. L.; Zhu, Z. J Am Soc Mass Spectrom 2016.

(4) Hua, X.; Marshall, M. J.; Xiong, Y. J.; Ma, X.; Zhou, Y. F.; Tucker, A. E.; Zhu, Z. H.; Liu, S. Q.; Yu, X. Y. Biomicrofluidics 2015, 9, 031101.

(5) Nygren, H.; Hagenhoff, B.; Malmberg, P.; Nilsson, M.; Richter, K. Microsc Res Tech 2007, 70, $969-974$.

(6) Weng, L. T.; Poleunis, C.; Bertrand, P.; Carlier, V.; Sclavons, M.; Franquinet, P.; Legras, R. J Adhes Sci Technol 1995, 9, 859-871.

(7) Ni, M.; Ratner, B. D. Surf Interface Anal 2008, 40, 1356-1361.

(8) Roberson, S. V.; Fahey, A. J.; Sehgal, A.; Karim, A. Appl Surf Sci 2002, 200, 150-164.

(9) Cizaire, L.; Martin, J. M.; Le Mogne, T.; Gresser, E. Colloid Surface A 2004, 238, 151-158.

(10) Briggs, D.; Brewis, D. M.; Dahm, R. H.; Fletcher, I. W. Surf Interface Anal 2003, 35, 156-167.

(11) Zeng, X. M.; Weng, L. T.; Li, L.; Chan, C. M.; Liu, S. Y.; Jiang, M. Surf Interface Anal 2001, 31, 421-428.

(12) Wu, L. G.; Lu, X. H.; Kulp, K. S.; Knize, M. G.; Berman, E. S. F.; Nelson, E. J.; Felton, J. S.; Wu, K. J. J. Int J Mass Spectrom 2007, 260, 137-145.

(13) Lim, Y. B.; Turpin, B. J. Atmos Chem Phys 2015, 15, 12867-12877.

(14) Liu, M.; Wang, H.; Griffiths, M. W. J Appl Microbiol 2007, 103, 2174-2184.

(15) Chang, C. Y.; Krishnan, T.; Wang, H.; Chen, Y.; Yin, W. F.; Chong, Y. M.; Tan, L. Y.; Chong, T. M.; Chan, K. G. Sci Rep-Uk 2014, 4.

(16) Lanni, E. J.; Masyuko, R. N.; Driscoll, C. M.; Aerts, J. T.; Shrout, J. D.; Bohn, P. W.; Sweedler, J. V. Anal Chem 2014, 86, 9139-9145.

(17) Lepine, F.; Milot, S.; Deziel, E.; He, J. X.; Rahme, L. G. J Am Soc Mass Spectr 2004, 15, 862-869.

(18) Lanni, E. J.; Masyuko, R. N.; Driscoll, C. M.; Dunham, S. J. B.; Shrout, J. D.; Bohn, P. W.; Sweedler, J. V. Anal Chem 2014, 86, 10885-10891.

(19) Timko, M. T.; Yu, Z. H.; Kroll, J. H.; Jayne, J. T.; Worsnop, D. R.; Miake-Lye, R. C.; Onasch, T. B.; Liscinsky, D.; Kirchstetter, T. W.; Destaillats, H.; Holder, A. L.; Smith, J. D.; Wilson, K. R. Aerosol Sci Tech 2009, 43, 855-865.

(20) Easton, M. P.; Labatete-Goeppinger, A. C.; Fowler, J. D.; Liu, D. L. Proc Spie 2014, 9196.

(21) O'Neill, E.; Harrington, D.; Allison, J. Anal Bioanal Chem 2009, 394, 2029-2038.

(22) Medard, N.; Poleunis, C.; Eynde, X. V.; Bertrand, P. Surf Interface Anal 2002, 34, 565-569.

(23) Kuo, T. M.; Manthey, L. K.; Hou, C. T. J Am Oil Chem Soc 1998, 75, 875-879.

(24) Wang, H. L.; Brattstrom, O.; Brakefield, P. M.; Francke, W.; Lofstedt, C. J Chem Ecol 2014, 40, $549-559$.

(25) Marsili, E.; Baron, D. B.; Shikhare, I. D.; Coursolle, D.; Gralnick, J. A.; Bond, D. R. P Natl Acad Sci USA 2008, 105, 3968-3973.

(26) Uyakul, D.; Isobe, M.; Goto, T. Bioorg Chem 1989, 17, 454-460.

(27) Xu, F.; Song, X. N.; Sheng, G. P.; Luo, H. W.; Li, W. W.; Yao, R. S.; Yu, H. Q. Sep Purif Technol 2015, 142, 18-24.

(28) Petrovic, M.; Barcelo, D. Anal Chem 2000, 72, 4560-4567.

(29) Keune, K.; Hoogland, F.; Boon, J. J.; Peggie, D.; Higgitt, C. Int J Mass Spectrom 2009, 284, 22-34.

(30) Amaya, K. R.; Sweedler, J. V.; Clayton, D. F. Journal of Neurochemistry 2011, 118, 499-511.

(31) Field, J. A.; Miller, D. J.; Field, T. M.; Hawthorne, S. B.; Giger, W. Anal Chem 1992, 64, 3161-3167.

(32) Ahmida, H. S. M.; Bertucci, P.; Franzo, L.; Massoud, R.; Cortese, C.; Lala, A.; Federici, G. Journal of Chromatography B-Analytical Technologies in the Biomedical and Life Sciences 2006, 842, 43-47.

(33) Zhuo, R. X.; Du, B.; Lu, Z. R. Journal of controlled release : official journal of the Controlled Release Society 1999, 57, 249-257.

(34) Meng, H.; Chen, Z. K.; Yu, W. L.; Pei, J.; Liu, X. L.; Lai, Y. H.; Huang, W. Synthetic Met 1999, $100,297-301$.

(35) Keune, K.; Boon, J. J. Surf Interface Anal 2004, 36, 1620-1628.

(36) Petrovic, M.; Barcelo, D. Anal Chem 2000, 72, 4560-4567.

(37) Leefmann, T.; Heim, C.; Kryvenda, A.; Siljestrom, S.; Sjovall, P.; Thiel, V. Organic Geochemistry 2013, 57, 23-33.

(38) Leefmann, T.; Heim, C.; Siljestrom, S.; Blumenberg, M.; Sjovall, P.; Thiel, V. Rapid Communications in Mass Spectrometry 2013, 27, 565-581.

(39) Qu, J.; Wang, Y. M.; Luo, G. A.; Wu, Z. P. J Chromatogr A 2001, 928, 155-162.

(40) Passarelli, M. K.; Winograd, N. Bba-Mol Cell Biol L 2011, 1811, 976-990.

(41) Hearn, M. J.; Briggs, D. Surf Interface Anal 1991, 17, 421-\&. 


\section{Supporting Information}

(42) Cillero-Pastor, B.; Eijkel, G.; Kiss, A.; Blanco, F. J.; Heeren, R. M. A. Anal Chem 2012, 84, 8909-8916.

(43) Aubriet, F.; Poleunis, C.; Bertrand, P. Journal of Mass Spectrometry 2001, 36, 641-651.

(44) Naithani, S.; Schaubroeck, D.; Vercammen, Y.; Mandamparambil, R.; Yakimets, I.; Van Vaeck, L.; Van Steenberge, G. Appl Surf Sci 2013, 280, 504-511.

(45) Hua, X.; Yu, X. Y.; Wang, Z. Y.; Yang, L.; Liu, B. W.; Zhu, Z. H.; Tucker, A. E.; Chrisler, W. B.; Hill, E. A.; Thevuthasan, T.; Lin, Y. H.; Liu, S. Q.; Marshall, M. J. Analyst 2014, 139, 1609-1613.

(46) Mas, S.; Touboul, D.; Brunelle, A.; Aragoncillo, P.; Egido, J.; Laprevote, O.; Vivanco, F. Analyst 2007, 132, 24-26.

(47) Ceglarek, U.; Efer, J.; Schreiber, A.; Zwanziger, E.; Engewald, W. Fresen J Anal Chem 1999, 365, 674-681.

(48) Barco, M.; Planas, C.; Palacios, O.; Ventura, F.; Rivera, J.; Caixach, J. Anal Chem 2003, 75, $5129-5136$.

(49) Mitchell, R.; Carr, C. M.; Parfitt, M.; Vickerman, J. C.; Jones, C. Cellulose 2005, 12, 629-639.

(50) Ryan, R. P.; Dow, J. M. Microbiol-Sgm 2008, 154, 1845-1858.

(51) Vickerman, J. C. Analyst 2011, 136, 2199-2217.

(52) Chandra, S.; Smith, D. R.; Morrison, G. H. Anal Chem 2000, 72, 104a-114a.

(53) McArthur, S. L.; Wagner, M. S.; Hartley, P. G.; McLean, K. M.; Griesser, H. J.; Castner, D. G. Surf Interface Anal 2002, 33, 924-931.

(54) Bruuning, C.; Hellweg, S.; Dambach, S.; Lipinsky, D.; Arlinghaus, H. F. Surf Interface Anal 2006, 38, 191193.

(55) Tyler, B. J.; Bruening, C.; Rangaranjan, S.; Arlinghaus, H. F. Biointerphases 2011, 6, 135-141.

(56) Groenewold, G. S.; Ingram, J. C.; McLing, T.; Gianotto, A. K.; Avci, R. Anal Chem 1998, 70, $534-539$.

(57) Ali, N.; Kousar, Y.; Okpalugo, T. I.; Singh, V.; Pease, M.; Ogwu, A. A.; Gracio, J.; Titus, E.; Meletis, E. I.; Jackson, M. J. Thin Solid Films 2006, 515, 59-65.

(58) Bokesch, H. R.; Pannell, L. K.; McKee, T. C.; Boyd, M. R. Tetrahedron Lett 2000, 41, 6305-6308.

(59) Lahann, J.; Balcells, M.; Rodon, T.; Lee, J.; Choi, I. S.; Jensen, K. F.; Langer, R. Langmuir 2002, 18, 36323638 .

(60) Duretz, B.; Schreiber, A.; Sakuma, T.; Sasaki, T.; Gamble, T.; Weinmann, W. Therapeutic Drug Monitoring 2007, 29, 498-498.

(61) Twomey, K. B.; O'Connell, O. J.; McCarthy, Y.; Dow, J. M.; O'Toole, G. A.; Plant, B. J.; Ryan, R. P. Isme J 2012, 6, 939-950.

(62) Reichlmaier, S.; Hammond, J. S.; Hearn, M. J.; Briggs, D. Surf Interface Anal 1994, 21, 739-746.

(63) Vaida, V. Journal of Chemical Physics 2011, 135.

(64) Verlet, J. R. R.; Bragg, A. E.; Kammrath, A.; Cheshnovsky, O.; Neumark, D. M. Science 2005, 307, 93-96.

(65) Sosnik, A.; Sodhi, R. N. S.; Brodersen, P. M.; Sefton, M. V. Biomaterials 2006, 27, 2340-2348.

(66) Bruny, G.; Eden, S.; Feil, S.; Fillol, R.; El Farkh, K.; Harb, M. M.; Teyssier, C.; Ouaskit, S.; Abdoul-Carime, H.; Farizon, B.; Farizon, M.; Mark, T. D. Rev Sci Instrum 2012, 83.

(67) Gianotto, A. K.; Hodges, B. D. M.; Benson, M. T.; Harrington, P. D.; Appelhans, A. D.; Olson, J. E.; Groenewold, G. S. J Phys Chem A 2003, 107, 5948-5955.

(68) Dizdaroglu, M.; Nackerdien, Z.; Chao, B. C.; Gajewski, E.; Rao, G. Archives of biochemistry and biophysics 1991, 285, 388-390.

(69) Karrasch, M.; Borner, G.; Enssle, M.; Thauer, R. K. Eur J Biochem 1990, 194, 367-372. 